Article

\title{
An Improved Mixed AC/DC Power Flow Algorithm in Hybrid AC/DC Grids with MT-HVDC Systems
}

\author{
Fazel Mohammadi ${ }^{1}{ }^{*}$, Gholam-Abbas Nazri ${ }^{2}$ and Mehrdad Saif ${ }^{1}$ \\ 1 Electrical and Computer Engineering (ECE) Department, University of Windsor, Windsor, ON N9B 1K3, \\ Canada \\ 2 Electrical and Computer Engineering, Wayne State University, Detroit, MI 48202, USA \\ * Correspondence: fazel@uwindsor.ca or fazel.mohammadi@ieee.org
}

Received: 30 November 2019; Accepted: 19 December 2019; Published: 31 December 2019

check for updates

\begin{abstract}
One of the major challenges on large-scale Multi-Terminal High Voltage Direct Current (MT-HVDC) systems is the steady-state interaction of the hybrid AC/DC grids to achieve an accurate Power Flow (PF) solution. In PF control of MT-HVDC systems, different operational constraints, such as the voltage range, voltage operating region, Total Transfer Capability (TTC), transmission reliability margin, converter station power rating, etc. should be considered. Moreover, due to the nonlinear behavior of MT-HVDC systems, any changes (contingencies and/or faults) in the operating conditions lead to a significant change in the stability margin of the entire or several areas of the hybrid AC/DC grids. As a result, the system should continue operating within the acceptable limits and deliver power to the non-faulted sections. In order to analyze the steady-state interaction of the large-scale MT-HVDC systems, an improved mixed AC/DC PF algorithm for hybrid AC/DC grids with MT-HVDC systems considering the operational constraints is developed in this paper. To demonstrate the performance of the mixed AC/DC PF algorithm, a five-bus AC grid with a three-bus MT-HVDC system and the modified IEEE 39-bus test system with two four-bus MT-HVDC systems (in two different areas) are simulated in MATLAB software and different cases are investigated. The obtained results show the accuracy, robustness, and effectiveness of the improved mixed AC/DC PF algorithm for operation and planning studies of the hybrid A/DC grids.
\end{abstract}

Keywords: improved mixed AC/DC power flow; multi-terminal high voltage direct current (MT-HVDC) systems; operational constraints; voltage-sourced converter (VSC)-high voltage direct current (HVDC) station

\section{Introduction}

Due to the recent developments in the power electronics technology, Voltage-Sourced Converter (VSC)-High Voltage Direct Current (HVDC) systems have solved the problem of bidirectional Power Flow (PF) in hybrid AC/DC grids [1-3]. MT-HVDC systems are capable of controlling the active and reactive power, independently. One of the important considerations to control the HVDC systems is that the $V_{D C}$-control and $P$-control VSC-HVDC stations should be capable of operating in inverter and rectifier modes, respectively [4-6]. The main purpose of applying different control strategies in MT-HVDC systems is to achieve a precise and secure control mode for MT-HVDC systems without violating the operational constraints. For stable operation and active and reactive PF, MT-HVDC systems need to maintain the DC voltage and frequency within the operating limits [1-4].

\subsection{AC/DC Power Flow for Hybrid AC/DC Grids with MT-HVDC Systems}

There have been some relevant surveys about power system operation considering PF problem solutions $[7,8]$. In traditional AC systems, the PF can be controlled through three hierarchical levels 
which are, HLI (generation level), HLII (generation and transmission levels), and HLIII (generation, transmission, and distribution levels). Considering hierarchical levels and the market analysis, the Independent Electricity System Operator (IESO) can only control the generated power on the power system, and based on that, control the PF to the next hierarchical level(s). The integration of MT-HVDC systems to the existing AC grids leads to increasing the region of controllability of the hybrid AC/DC grids. This fact is due to the capability of controlling active and reactive PF by each converter station in MT-HVDC systems $[1,4,9]$. Therefore, MT-HVDC systems can change the PF patterns, and from the IESO and Transmission System Operator (TSO) perspectives, these changes in the pattern may cause significant issues in hybrid AC/DC grids. Moreover, due to the temporary or permanent outages of the components in hybrid AC/DC grids, the dynamic behavior of nonlinear components can change the $\mathrm{PF}$ and enhance the risk of instability in the entire hybrid AC/DC grids [9-11].

\subsection{Solving the Power Flow Problem for Hybrid AC/DC Grids with MT-HVDC Systems}

The unified method and sequential method are the two well-known methods to solve the PF problem for hybrid AC/DC grids with MT-HVDC systems.

\subsubsection{The Unified Method}

The unified method solves the PF problem for the entire hybrid AC/DC grids using a modified Jacobian matrix [12-14], where all the AC and DC variables, such as the impact of DC links in the Jacobian matrix, can be calculated in each iteration process. Many techniques are proposed and developed to improve the efficiency of the unified method, but the main drawback of those methods is neglecting the impact of droop parameters' settings on the AC/DC PF [12-14]. Another issue of the unified method to solve the PF problem is that it needs an alternation of the extension of an existing AC PF.

\subsubsection{The Sequential Method}

The sequential method solves the AC/DC PF equations sequentially, one after another, in each iteration $[15,16]$. The main advantage of the sequential method is to make the solution easy to combine the DC PF to the AC PF solution, and it can be implemented easily when the extension of an existing AC PF is needed. In [17], a numerical method based on the Newton-Raphson algorithm to calculate the converter station losses is proposed. A detailed steady-state model of the converter station to solve the AC/DC PF problem sequentially based on the Newton-Raphson technique considering converter station losses and reference power is proposed in $[18,19]$. An algorithm with per-unit conversion and changing the bus numbers to simplify solving the PF problem when multiple DC lines and converter station outages occur is developed in [18]. The main drawback of this method is neglecting the AC grid connection in the problem. In [20], a detailed model of the converter station with AC/DC PF equations, including converter station losses, for solving the PF sequentially is proposed. In [21], a method to solve the PF problem through the Gauss-Seidel method is developed.

\subsection{AC/DC Power Flow for MT-HVDC Systems Considering Droop Parameters}

Controlling the droop parameters in MT-HVDC systems has a significant impact on the PF of AC and DC grids after an outage [4]. In [16], the concept of distributed DC voltage control with the droop parameters on the PF problem in MT-HVDC systems is considered. In [22], a methodology to determine the mean voltage instead of a single slack converter station in MT-HVDC systems to solve the DC PF is proposed. By interconnecting MT-HVDC systems to the large-scale AC grids, solving the AC/DC PF goes through a complicated process, and a combined solution of AC/DC PF considering all the system's variables and constraints is required.

To address all the above-mentioned challenges, a mixed AC/DC PF algorithm for hybrid AC/DC grids with MT-HVDC systems is proposed in this paper. The proposed strategy is a fast and accurate method, which is capable of optimizing the AC/DC PF calculations. Except for the high accuracy and 
optimized performance, considering all operational constraints and control objectives of the integration of MT-HVDC systems into the large-scale AC grids is the other contribution of this paper. The calculated results by the mixed AC/DC PF problem can be used for the planning, scheduling, state estimation, small-signal stability analyses. The mixed AC/DC PF algorithm is applied to a five-bus AC grid with a three-bus MT-HVDC system and the modified IEEE 39-bus test system with two four-bus MT-HVDC systems (in two different areas) which are simulated in MATLAB software. To check the performance of the mixed AC/DC PF algorithm, different cases are considered. The obtained results show the accuracy, robustness, and effectiveness of the mixed AC/DC PF algorithm.

\section{Principles of Power Flow in Power Systems}

\subsection{AC Grid Power Flow}

The main objective of the AC PF is to determine the magnitude and angle at each bus of the AC grids. In order to analyze the AC PF in power systems, the following assumptions are considered.

- The AC transmission networks have fast dynamics compared to the other components. In this regard, AC transmission networks can be represented by algebraic equations.

- Each transmission line and transformer is modeled by an equivalent $\pi$ model.

- The power in AC grids is balanced.

- The positive sequence parameters on a per-phase basis are assumed.

Accordingly, the network equations can be written as follows:

$$
\left[\begin{array}{c}
\bar{I}_{1} \\
\bar{I}_{2} \\
\vdots \\
\bar{I}_{i} \\
\vdots \\
\bar{I}_{n_{A C}}
\end{array}\right]=\left[\begin{array}{cccccc}
\bar{Y}_{11} & \bar{Y}_{12} & \ldots & \bar{Y}_{1 i} & \ldots & \bar{Y}_{1 n_{A C}} \\
\bar{Y}_{21} & \bar{Y}_{22} & \ldots & \bar{Y}_{2 i} & \ldots & \bar{Y}_{2 n_{A C}} \\
\vdots & \vdots & \vdots & \vdots & \vdots & \vdots \\
\bar{Y}_{i 1} & \bar{Y}_{i 2} & \cdots & \bar{Y}_{i i} & \ldots & \bar{Y}_{i n_{A C}} \\
\vdots & \vdots & \vdots & \vdots & \vdots & \vdots \\
\bar{Y}_{n_{A C} 1} & \bar{Y}_{n_{A C} 2} & \cdots & \bar{Y}_{n_{A C} i} & \cdots & \bar{Y}_{n_{A C} n_{A C}}
\end{array}\right]\left[\begin{array}{c}
\bar{V}_{1} \\
\bar{V}_{2} \\
\vdots \\
\bar{V}_{i} \\
\vdots \\
\bar{V}_{n_{A C}}
\end{array}\right]
$$

where $\bar{I}_{i}$ and $\bar{V}_{i}=V_{i} e^{j \theta_{i}}$ are the injected current and voltage at the $i$ th node, $\bar{Y}_{i i}$ is the self-admittance at the $i$ th node, $\bar{Y}_{i j}$ is the mutual admittance between nodes $i$ and $j$, and $n_{A C}$ represents the total number of buses in the AC grid.

The voltage of each node can be used for solving the equation of $[\bar{I}]-\left[\overline{Y_{b u s}}\right][\bar{V}]=0$. The injected current at the $i$ th node is related to the injected power and bus voltage at that node and it can be calculated as follows:

$$
\bar{I}_{i}=\frac{P_{i}-j Q_{i}}{\bar{V}_{i}^{*}}
$$

Considering the operational constraints in power systems, the PF problem becomes nonlinear, and the nodes in AC grids can be classified into four types:

- $\quad$ Slack bus where the voltage magnitude $\left(V_{i}\right)$ and angle $\left(\theta_{i}\right)$ are determined.

- PV bus where the active power $\left(P_{i}\right)$ injected to the grid and the voltage magnitude $\left(V_{i}\right)$ are known.

- $\quad P Q$ bus where the active power and reactive power $\left(Q_{i}\right)$ injected into the grid are known.

- MT-HVDC Point of Common Coupling (PCC) bus where different constraints based on the control mode of each converter station are applied.

From Equation (1), the injected current at the ith node (without considering the PCC buses of MT-HVDC systems) can be written as follows:

$$
\bar{I}_{i}=\sum_{j=1}^{n_{A C}} \overline{Y_{i j} V_{j}}
$$


Therefore, the active and reactive power injected to the grid can be derived as follows:

$$
\begin{aligned}
P_{i} & =\sum_{j=1}^{n_{A C}} V_{i} V_{j}\left[G_{i j} \cos \left(\theta_{i}-\theta_{j}\right)+B_{i j} \sin \left(\theta_{i}-\theta_{j}\right)\right] \\
Q_{i} & =\sum_{j=1}^{n_{A C}} V_{i} V_{j}\left[G_{i j} \sin \left(\theta_{i}-\theta_{j}\right)-B_{i j} \cos \left(\theta_{i}-\theta_{j}\right)\right]
\end{aligned}
$$

where $\bar{Y}_{i j}=G_{i j}+j B_{i j}$.

It should be noted that a generator can be either a slack bus or a PV bus, and a load can be a PQ bus with known active and reactive power.

\subsection{MT-HVDC Systems Power Flow}

The main aim of MT-HVDC systems PF is to determine the DC voltage magnitude at each converter station and the PF within the DC grid. Considering $n$ converter stations in MT-HVDC systems, the following items should be considered to derive the PF equations for the $i$ th converter station of MT-HVDC systems.

- Interface of the converter station with the AC grid

- AC side of the converter station

- Interface of the converter station AC and DC sides

- $\quad$ DC side of MT-HVDC systems

- Control modes of the converter station

\subsubsection{Interface of the Converter Station with the AC Grid}

The PF equations of at the PCC bus of the $i$ th converter station interfacing with the AC grid is as follows:

$$
\begin{aligned}
& P_{g i}=\sum_{j=1}^{n_{A C}} V_{g i} V_{j}\left[G_{i j} \cos \left(\theta_{g i}-\theta_{j}\right)+B_{i j} \sin \left(\theta_{g i}-\theta_{j}\right)\right] \\
& Q_{g i}=\sum_{j=1}^{n_{A C}} V_{g i} V_{j}\left[G_{i j} \sin \left(\theta_{g i}-\theta_{j}\right)-B_{i j} \cos \left(\theta_{g i}-\theta_{j}\right)\right]
\end{aligned}
$$

where $\bar{V}_{g i}=V_{g i} e^{j \theta_{g i}}$.

\subsubsection{AC Side of the Converter Station}

The PF equations of at the PCC bus of the $i$ th converter station for the asymmetric bipolar MT-HVDC systems is as follows:

$$
\begin{gathered}
P_{g i}-P_{g i}^{p}-P_{g i}^{n}=0 \\
Q_{g i}-Q_{g i}^{p}-Q_{g i}^{n}=0
\end{gathered}
$$

where $P_{g i}^{p}, P_{g i}^{n} Q_{g i}^{p}$ and $Q_{g i}^{n}$ are the active and reactive power of the positive and negative poles of the $i$ th converter station, respectively.

Therefore, the apparent power at the PCC bus of the ith converter station by the positive and negative poles can be written as follows:

$$
\begin{gathered}
S_{g i}^{p}=\bar{V}_{g i} \bar{I}_{g i}^{p^{*}}=P_{g i}^{p}+j Q_{g i}^{p} \\
S_{g i}^{n}=\bar{V}_{g i} \bar{I}_{g i}^{n^{*}}=P_{g i}^{n}+j Q_{g i}^{n}
\end{gathered}
$$


Applying KCL at the PCC bus of the $i$ th converter station leads to deriving the following equation.

$$
\bar{I}_{g i}=\bar{I}_{g i}^{p}+\bar{I}_{g i}^{p}
$$

Using KVL between the PCC bus and the converter terminal of the $i$ th converter station leads to deriving the following equations.

$$
\begin{aligned}
& \bar{I}_{g i}^{p}=\frac{\bar{V}_{t i}^{p}-\bar{V}_{g i}}{Z_{i}^{p}}=\left(\bar{V}_{t i}^{p}-\bar{V}_{g i}\right)\left(G_{i}^{p}+j B_{i}^{p}\right) \\
& \bar{I}_{g i}^{n}=\frac{\bar{V}_{t i}^{n}-\bar{V}_{g i}}{Z_{i}^{n}}=\left(\bar{V}_{t i}^{n}-\bar{V}_{g i}\right)\left(G_{i}^{n}+j B_{i}^{n}\right)
\end{aligned}
$$

where $Z_{i}^{p}=R_{i}^{p}+j X_{i}^{p}$ and $Z_{i}^{n}=R_{i}^{n}+j X_{i}^{n}$. Also, $\bar{V}_{t i}^{p}=V_{t i}^{p} e^{j \theta_{t i}^{p}}$ and $\bar{V}_{t i}^{n}=V_{t i}^{n} e^{j \theta_{t i}^{n}}$ are the voltage of the positive and negative pole converter terminal, respectively.

Substituting Equations (13) and (14) into Equations (10) and (11), and also separating the active and reactive power, the following equations are obtained.

$$
\begin{gathered}
P_{g i}^{p}=V_{g i}\left[-V_{g i} G_{i}^{p}+V_{t i}^{p}\left\{G_{i}^{p} \cos \left(\theta_{g i}-\theta_{t i}^{p}\right)+B_{i}^{p} \sin \left(\theta_{g i}-\theta_{t i}^{p}\right)\right\}\right] \\
Q_{g i}^{p}=V_{g i}\left[V_{g i} B_{i}^{p}+V_{t i}^{p}\left(G_{i}^{p} \sin \left(\theta_{g i}-\theta_{t i}^{p}\right)-B_{i}^{p} \cos \left(\theta_{g i}-\theta_{t i}^{p}\right)\right\}\right] \\
P_{g i}^{n}=V_{g i}\left[-V_{g i} G_{i}^{n}+V_{t i}^{n}\left\{G_{i}^{n} \cos \left(\theta_{g i}-\theta_{t i}^{n}\right)+B_{i}^{n} \sin \left(\theta_{g i}-\theta_{t i}^{n}\right)\right\}\right] \\
Q_{g i}^{n}=V_{g i}\left[V_{g i} B_{i}^{n}+V_{t i}^{n}\left\{G_{i}^{n} \sin \left(\theta_{g i}-\theta_{t i}^{n}\right)-B_{i}^{n} \cos \left(\theta_{g i}-\theta_{t i}^{n}\right)\right\}\right]
\end{gathered}
$$

\subsubsection{Interface of the Converter Station AC and DC Sides}

Regardless of the converter station switching losses, the active power balance between converter station AC and DC sides is as follows:

$$
\begin{aligned}
& P_{t i}^{p}=V_{D C i}^{p} I_{D C i}^{p} \\
& P_{t i}^{n}=V_{D C i}^{n} I_{D C i}^{n}
\end{aligned}
$$

where $V_{D C i^{\prime}}^{p} I_{D C i^{\prime}}^{p} V_{D C i^{\prime}}^{n}$ and $I_{D C i}^{n}$ are the DC bus voltage and current of the positive and negative pole of the $i$ th converter station.

Also, the active power at the AC-side terminal of the positive and negative pole of the converter stations can be written as follows:

$$
\begin{aligned}
& P_{t i}^{p}=V_{t i}^{p}\left[V_{t i}^{p} G_{i}^{p}-V_{g i}\left\{G_{i}^{p} \cos \left(\theta_{t i}^{p}-\theta_{g i}\right)+B_{i}^{p} \sin \left(\theta_{t i}^{p}-\theta_{g i}\right)\right\}\right] \\
& P_{t i}^{n}=V_{t i}^{n}\left[V_{t i}^{n} G_{i}^{n}-V_{g i}\left\{G_{i}^{n} \cos \left(\theta_{t i}^{n}-\theta_{g i}\right)+B_{i}^{n} \sin \left(\theta_{t i}^{n}-\theta_{g i}\right)\right\}\right]
\end{aligned}
$$

\subsubsection{Side of MT-HVDC Systems}

When the current flowing through the DC-bus capacitor is zero, $I_{D C i}^{p}=I_{L i}^{p}$ and $I_{D C i}^{n}=I_{L i}^{n}$, where $I_{L i}^{p}$ and $I_{L i}^{n}$ are the current of the positive and negative DC link connected to the $i$ th $h$ converter station. 
The injected current can be determined by the conductance matrix of the DC grid. The DC current injection at the positive and negative poles of the DC bus is as follows:

$$
\left[\begin{array}{c}
-I_{D C 1}^{p} \\
\vdots \\
-I_{D C i}^{p} \\
\vdots \\
-I_{D C N}^{p} \\
-I_{D C 1}^{n} \\
\vdots \\
-I_{D C i}^{n} \\
\vdots \\
-I_{D C N}^{n}
\end{array}\right]=\left[\begin{array}{cccccccccc}
G_{p p 11} & \cdots & G_{p p 1 i} & \cdots & G_{p p 1 N} & G_{p n 11} & \cdots & G_{p n 1 i} & \cdots & G_{p n 1 N} \\
\vdots & \vdots & \vdots & \vdots & \vdots & \vdots & \vdots & \vdots & \vdots & \vdots \\
G_{p p i 1} & \cdots & G_{p p i i} & \cdots & G_{p p i N} & G_{p n i 1} & \cdots & G_{p n i i} & \cdots & G_{p n i N} \\
\vdots & \vdots & \vdots & \vdots & \vdots & \vdots & \vdots & \vdots & \vdots & \vdots \\
G_{p p N 1} & \cdots & G_{p p N i} & \cdots & G_{p p N N} & G_{p n N 1} & \cdots & G_{p n N i} & \cdots & G_{p n N N} \\
G_{n p 11} & \cdots & G_{n p 1 i} & \cdots & G_{n p 1 N} & G_{n n 11} & \cdots & G_{n n 1 i} & \cdots & G_{n p 1 N} \\
\vdots & \vdots & \vdots & \vdots & \vdots & \vdots & \vdots & \vdots & \vdots & \vdots \\
G_{n p i 1} & \cdots & G_{n p i i} & \cdots & G_{n p i N} & G_{n n i 1} & \cdots & G_{n n i i} & \cdots & G_{n p i N} \\
\vdots & \vdots & \vdots & \vdots & \vdots & \vdots & \vdots & \vdots & \vdots & \vdots \\
G_{n p N 1} & \cdots & G_{n p N i} & \cdots & G_{n p N N} & G_{n n N 1} & \cdots & G_{n n N i} & \cdots & G_{n p N N}
\end{array}\right]\left[\begin{array}{c}
V_{D C 1}^{p} \\
\vdots \\
V_{D C i}^{p} \\
\vdots \\
V_{D C N}^{p} \\
V_{D C 1}^{n} \\
\vdots \\
V_{D C i}^{n} \\
\vdots \\
V_{D C N}^{n}
\end{array}\right]
$$

Hence, the injected current at the $i$ th bus in the DC side is as follows:

$$
\begin{gathered}
I_{D C i}^{p}=-\sum_{j=1}^{N}\left(G_{p p j i} V_{D C j}^{p}+G_{p n j i} V_{D C j}^{n}\right) \\
I_{D C i}^{n}=-\sum_{j=1}^{N}\left(G_{n p j i} V_{D C j}^{p}+G_{n n j i} V_{D C j}^{n}\right)
\end{gathered}
$$

where $N$ represents the total number of buses in the DC grid. In addition, $G_{p p j i}, G_{n n j i}, G_{p n j i}$, and $G_{n p j i}$ are the conductance of the positive and negative DC link between nodes $i$ and $j$, respectively.

\subsubsection{Control Modes of the Converter Station}

There are different modes of operation for each converter station in MT-HVDC systems [4], and based on them, the steady-state equations in the form of equality constraints can be obtained. Also, there are some inequality constraints due to the limits, which are imposed by the converter station voltage and current ratings, as follows:

$$
\begin{gathered}
V_{D C i}^{\min } \leq V_{D C i} \leq V_{D C i}^{\max } \\
V_{i}^{\min } \leq V_{i} \leq V_{i}^{\max } \\
I_{g i} \leq I_{g i}^{\max } \\
I_{D C i} \leq I_{D C i}^{\max }
\end{gathered}
$$

\section{Mixed AC/DC Power Flow Algorithm}

The mixed AC/DC PF algorithm, which is an improved sequential AC/DC PF algorithm [19], can be used to obtain the initial operating points to analyze the dynamics of the hybrid AC/DC grids by solving the AC and DC PF sequentially and keeping both the converter station power and voltage at each node constant.

In order to implement the mixed AC/DC PF algorithm, the per-unit conversion should be performed for the entire system. Each converter station is connected to both AC and DC grids. The AC side of the converter station is modeled by a voltage source connected to the AC bus through a phase reactor $\left(Z_{C}=R_{C}+j \omega L_{C}\right)$, a capacitor $\left(Z_{F}=\frac{-j}{\omega C}\right)$, and a transformer $\left(Z_{T R}=R_{T R}+j \omega L_{T R}\right)$. Also, the DC side of the converter station is connected to the DC grid. The power losses at the converter 
station are considered as a quadratic function of the converter station Root Mean Square (RMS) AC current as follows [23]:

$$
P_{\text {Loss }}=a+b I+c I^{2}
$$

where $a, b$, and $c$ are the loss coefficients.

It should be noted that,

$$
P_{g i}+P_{D C i}+P_{\text {Lossi }}=0
$$

Assume an MT-HVDC system with $n$ converter stations and $n_{D C}$ DC lines. For AC/DC PF analysis, at least one converter station should be capable of controlling $V_{D C}$ (DC-slack bus) in the entire DC grids, and the rest of the converter stations control the active power. The detailed explanations of the mixed AC/DC PF are given as follows:

1. Start by an initial guess of the active power injected to the AC grid by the DC-slack converter station at the $l$ th iteration $\left(P_{g n_{s}}^{(l)}\right)$, where $n_{s}$ represents the index of the DC-slack bus.

2. Transform all converter stations which are connected to the $j$ th bus to PV or PQ buses based on their control modes (including droop-based control strategies) and solve the AC PF. At the lth iteration, the active power injected by all the non-slack converter stations is constant, while $P_{g n_{s}}^{(l)}$ changes.

3. Calculate the converter station losses using Equation (30) considering the active power injected by the $i$ th converter to the AC grid $\left(P_{g i}^{(l)}\right)$ and the active power injected by the $i$ th converter station to the DC grid $\left(P_{D C i}^{(l)}\right)$. In this step, the AC/DC connections and the converter stations' limits should be considered.

4. Solve the DC PF for the DC grid using the Newton-Raphson method. In this step, the DC-slack bus regulates the DC voltage $\left(V_{D C n_{s}}^{(0)}\right)$ initially, and the DC buses determine the active power injection $\left(P_{D C i}^{(l)}\right)$. Therefore, the DC voltage at each bus and also $P_{D C n_{s}}^{(l)}$ are calculated.

5. Compute DC-slack and droop buses iteration $(k)$. As a new value of $P_{g n_{s}}^{(l+1)}$ is calculated, considering the converter station losses,

I. Initialization: $P_{g n_{s}}^{(k=0)}=P_{g n_{s}}^{(l)}$.

II. Solve the branch $j, n_{s}-g, n_{s}$ considering $V_{j n s}, \theta_{j n s}, Q_{j n s}$, and $P_{g n_{s}}^{(k)}$ using the Newton-Raphson method.

III. Obtain the new value of $P_{g n_{s}}^{(k+1)}$ with $P_{D C n s}$ and $P_{\text {Lossns }}$ using Equations (30) and (31).

IV. If $\left|P_{g n_{s}}^{(k+1)}-P_{g n_{s}}^{(k)}\right|<\varepsilon$, stop the calculations. Otherwise, $k=k+1$ and return to step II. The output is $P_{j n_{s}}^{(k+1)}$.

6. Check the convergence criterion. If $\left|P_{g n_{s}}^{(k+1)}-P_{g n_{s}}^{(k)}\right|<\varepsilon$, stop the calculations. Otherwise, $k=k+1$ and return to step 1.

It should be noted that all linear and nonlinear variables are considered in the mixed AC/DC PF algorithm. Based on the topology of the hybrid AC/DC grids, the rating and length of each transmission line are determined. Also, the droop parameters and reference voltage have a direct impact on the reference power.

\section{Results and Discussions}

For validation and to demonstrate the performance of the mixed AC/DC PF algorithm, a five-bus AC grid with a three-bus MT-HVDC system is simulated in MATLAB software, as shown in Figure 1. The test system is composed of both AC and DC grids. The data of the system is provided in Tables 1-5. 


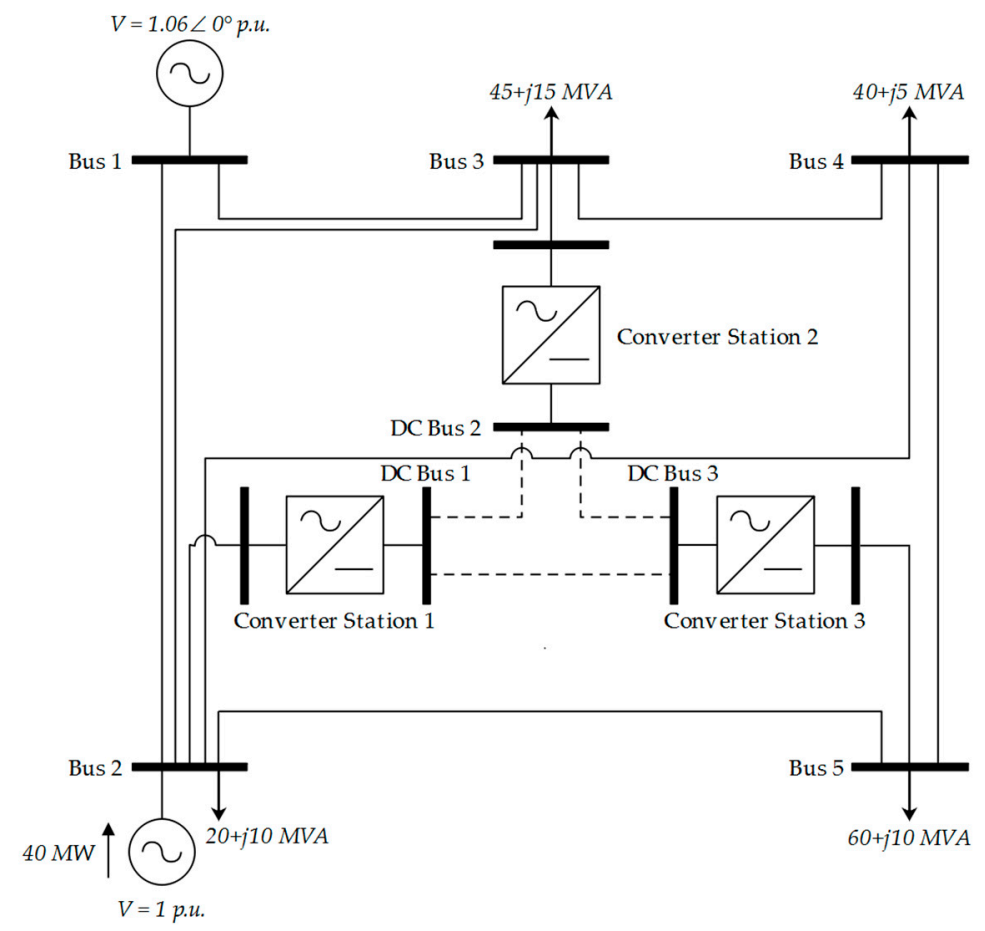

Figure 1. Single-line diagram of a three-bus MT-HVDC system.

Table 1. Parameters of the AC system.

\begin{tabular}{cccccccc}
\hline Bus & Type & $\boldsymbol{V}$ (p.u.) & $\boldsymbol{\theta}\left(^{\circ}\right)$ & $\boldsymbol{P}_{G}(\mathbf{M W})$ & $\boldsymbol{Q}_{G}$ (MVAR) & $\boldsymbol{P}_{\boldsymbol{D}}(\mathbf{M W})$ & $\boldsymbol{Q}_{\boldsymbol{D}}$ (MVAR) \\
\hline 1 & Slack & 1.060 & 0.00 & - & - & 0.00 & 0.00 \\
2 & $\mathrm{PV}$ & 1.000 & - & 40.00 & - & 20.00 & 10.00 \\
3 & $\mathrm{PQ}$ & - & - & - & - & 45.00 & 15.00 \\
4 & $\mathrm{PQ}$ & - & - & - & - & 40.00 & 5.00 \\
5 & $\mathrm{PQ}$ & - & - & - & - & 60.00 & 10.00 \\
\hline
\end{tabular}

Table 2. Parameters of the AC lines.

\begin{tabular}{cccccc}
\hline From & To & $\boldsymbol{R}$ (p.u.) & $\boldsymbol{X}$ (p.u.) & $\boldsymbol{B}$ (p.u.) & $\boldsymbol{S}_{\boldsymbol{N}}$ (MVA) \\
\hline 1 & 2 & 0.02 & 0.06 & 0.06 & 100 \\
1 & 3 & 0.08 & 0.24 & 0.05 & 100 \\
2 & 3 & 0.06 & 0.18 & 0.04 & 100 \\
2 & 4 & 0.06 & 0.18 & 0.04 & 100 \\
2 & 5 & 0.04 & 0.12 & 0.03 & 100 \\
3 & 4 & 0.01 & 0.03 & 0.02 & 100 \\
4 & 5 & 0.08 & 0.24 & 0.05 & 100 \\
\hline
\end{tabular}

Table 3. Parameters of the converter stations in per-unit.

\begin{tabular}{ccccccc}
\hline Converter Station & $S_{N}$ (MVA) & $\boldsymbol{R}_{T R}$ & $\boldsymbol{X}_{T R}$ & $\boldsymbol{B}_{\boldsymbol{F}}$ & $\boldsymbol{R}_{\boldsymbol{T R}}$ & $\boldsymbol{X}_{\boldsymbol{T R}}$ \\
\hline 1 & 100 & 0.0015 & 0.1121 & 0.0887 & 0.0001 & 0.1643 \\
2 & 100 & 0.0015 & 0.1121 & 0.0887 & 0.0001 & 0.1643 \\
3 & 100 & 0.0015 & 0.1121 & 0.0887 & 0.0001 & 0.1643 \\
\hline
\end{tabular}


Table 4. Power losses coefficients of the converter stations.

\begin{tabular}{ccccc}
\hline Converter Station & $\boldsymbol{a}$ & $\boldsymbol{b}$ & $\boldsymbol{c}_{\text {rec }}$ & $\boldsymbol{c}_{\text {inv }}$ \\
\hline 1 & 1.103 & 0.887 & 2.885 & 4.371 \\
2 & 1.103 & 0.887 & 2.885 & 4.371 \\
3 & 1.103 & 0.887 & 2.885 & 4.371 \\
\hline
\end{tabular}

Table 5. Parameters of the DC lines.

\begin{tabular}{ccccc}
\hline From & To & $\boldsymbol{R}_{\boldsymbol{D C}}$ (p.u.) & $\boldsymbol{V}_{\boldsymbol{D C}}(\mathbf{k V )}$ & $\boldsymbol{P}_{\boldsymbol{D C}}(\mathbf{M W})$ \\
\hline 1 & 2 & 0.0260 & 345 & 100 \\
1 & 3 & 0.0365 & 345 & 100 \\
2 & 3 & 0.0260 & 345 & 100 \\
\hline
\end{tabular}

The parameters of the converter stations for the PF calculations are as follows:

- Converter Station \#1: $P-Q$ control mode, $P_{g}=-60 \mathrm{MW}$ and $Q_{g}=-40 \mathrm{MVAR}$

- Converter Station \#2: $V_{D C}-V$ control mode, $V_{D C}=1$ p.u. and $V=1$ p.u.

- Converter Station \#3: $P-Q$ control mode, $P_{g}=35 \mathrm{MW}$ and $Q_{g}=5 \mathrm{MVAR}$

It should be noted that $S_{B}=100 \mathrm{MVA}, V_{D C_{B}}=345 \mathrm{kV}$, and $V_{B}=345 \mathrm{kV}$ are the base values for the hybrid AC/DC grids per-unit system. Also, the links between the two nodes on the DC grids are bipolar.

The simulations are accomplished using a laptop with the Intel Core i7-8550U processor at $1.80 \mathrm{GHz}$ clock speed and 12-GB of RAM.

\subsection{Case 1: AC Power Flow without MT-HVDC Systems}

In the first case, the AC PF calculations are performed without considering the DC system. The algorithm is converged in $0.44 \mathrm{~s}$ and in three iterations. Tables A1 and A2 show the results of AC PF without DC grids.

\subsection{Case 2: AC/DC Power Flow Considering MT-Systems with Constant Active Power and DC Voltage}

In the second case, the algorithm is applied to solve the AC/DC PF problem for the studied system considering that converter stations \#1 and \#3 are operated in constant $P$-mode and converter station $\# 2$ is operated in constant $V_{D C}$-mode. The algorithm is converged in $0.82 \mathrm{~s}$ and in three iterations. Tables A3-A7 show the obtained results of the mixed AC/DC PF calculations in Case 2.

Compared to Case 1, in this case, both the active and reactive PF on each line have decreased and accordingly, the total power losses are decreased. It is observed that the generator connected to bus 2 injects its maximum active power. In addition, compared to the previous case, the total injected reactive power by the two generators is decreased.

\subsection{Case 3: AC/DC Power Flow Considering Converter Station Outage}

In the third case, the impact of the converter station outage on the AC/DC PF results is analyzed. Tables A8-A11 demonstrate the results of PF calculations both in all AC and DC buses and in branches, in case of the outage of converter station \#1. Based on the obtained results, the converter station \#3 is capable of operating in constant $P$-mode. It should be noted that the algorithm is converged in $0.55 \mathrm{~s}$ and in three iterations.

In this case, the generator connected to bus 2 injects $40 \mathrm{MW}$ active power to the grid (maximum active power capability) and the total injected reactive power this generator is approximately tripled. The outage of converter station \#1 is led to an increase in the active and reactive PF of the AC lines. Therefore, the total power losses on the AC grids are increased. Due to the fact that converter station 
\#1 is not connected to the grid, the summation of the PF between the DC lines (1-2 and 1-3) is zero and consequently, the overall DC power losses are decreased.

\subsection{Case 4: AC/DC Power Flow Considering Droop Control Strategy and Converter Outage}

As stated in Section 1.3, the droop control strategy is an efficient way of controlling MT-HVDC systems that can improve the PF of the AC and DC grids after an outage. In the fourth case, it is assumed that all converter stations in the studied system are equipped with the droop controllers. Table A12 illustrates the detailed information of the droop settings for each converter station. Tables A13-A16 show the results of PF calculations both in all AC and DC buses and in branches in case of the outage of converter station \#1 and considering droop control settings provided in Table A12. It should be noticed that the algorithm is converged in $0.45 \mathrm{~s}$ and in three iterations.

Compared to Case 3, in this case, the contribution of the generator connected to bus 2 to power generation is decreased. As the outage of converter station \#1 is considered, compared to Case 3 , the PF on the DC lines is decreased. It should be noted that as the performance of each converter station is based on the pre-determined droop parameter, the PF on the DC lines should be in a way that the total power losses on the DC lines become zero.

\subsection{Case 5: AC/DC Power Flow Considering Changes of Droop Parameters and Converter Outage}

In the fifth case, to demonstrate the impact of changes in the droop parameters of each converter station, the same test as Case 4 is evaluated by changing the droop parameters as shown in Table A17. Tables A18-A21 depict the results of PF calculations both in all AC and DC buses and in branches in case of the outage of converter station \#1 and considering the changes in droop parameters according to Table A17. It is worth mentioning that the algorithm is converged in $0.43 \mathrm{~s}$ and in three iterations.

Changing the droop parameters is led to an increase in the total active power generated by the generator connected to bus 2 . As a consequence, there are some slight changes of the PF on the AC lines. However, compared to the previous case, those changes are negligible. In addition, changing the droop parameters is causes an increase in the DC PF on the DC lines (compared to Case 4), but as the converter station \#1 is not connected to the grid, the summation of the PF between the DC lines is equal to zero.

\subsection{Case 6: AC/DC Power Flow Considering Converter Station Limits and Converter Outage}

In the sixth case, the impact of the converter station limits on the AC/DC PF solution is investigated. When an active power set-point of a converter station equipped with the $P$ controller is outside of the $\mathrm{P}-\mathrm{Q}$ capability chart ( $\mathrm{P}-\mathrm{Q}$ capability chart shows the possible operation points.), the active power order should be reduced to comply with the predefined limit. Similarly, when a reactive power set-point of a converter station equipped with the $Q$ controller is outside of the P-Q capability chart, the reactive power order should be reduced to comply with the predefined limit, subject to not reaching the active power limit. In addition, when a converter station equipped with the $V_{D C}$-droop controller reaches its limit, the converter station should be set to a constant $P$ injection equal to the maximum active power limit of the converter station. In addition, when a converter station equipped with the $V$-droop controller reaches its limit, the converter station should be set to a constant $Q$ injection based on the predefined limit. For both converter stations equipped with the $Q$ controller and $V_{D C}$-droop controller, the priority is given to active power over reactive power, when enforcing the limits. It should be noted that however, all the DC-slack buses are disregarded from the analysis, they are rechecked at the end of AC/DC PF calculations.

According to the above explanations, it is assumed that the converter station \#1 reaches the reactive power limit and the converter station control is changed from constant $V$-mode to constant Q-mode. Tables A22-A25 illustrate the results of PF calculations in all both AC and DC buses and branches after enforcing the converter station's current and voltage limits. Meanwhile, the algorithm 
is converged in $0.60 \mathrm{~s}$ and in three iterations. After three iterations, the reactive power set-point of the converter station \#1 is changed from -40 MVAR to -36.25 MVAR.

To demonstrate the impact of changing the set-point of the converter station, the active power set-point of the converter station \#1 is set to $-130 \mathrm{MW}$ so that simultaneously both active and reactive power violate their limits. Tables A26-A29 show the results of PF calculations both in all AC and DC buses and in branches after changing the active power set-point of the converter station \#1 to $-130 \mathrm{MW}$. It should be noted that the algorithm is converged in $0.71 \mathrm{~s}$ and in three iterations.

After three iterations, the active and reactive power set-points of the converter station \#1 are changed from -130 MW to -121.21 MW, and -40 MVAR to -8.96 MVAR, respectively. In this case, the total active power generated by the generation units is increased (tangible changes are related to the one connected to bus 2). The overall power losses on the AC lines are decreased. As the converter station \#1 is connected to the grid and its set-points are reached their maximums limits, the active power losses (correspond to $\mathrm{ZI}^{2}$ ) are increased. Therefore, the PF on the DC lines is increased.

\subsection{Case 7: AC/DC Power Flow for the Large-Scale Hybrid AC/DC Grids with MT-HVDC Systems}

In order to validate and show the performance of the mixed AC/DC PF algorithm for the large-scale hybrid AC/DC grids with the integration of MT-HVDC system, the modified IEEE 39-bus test system with two four-bus MT-HVDC systems (with different colors) is simulated in MATLAB software, as shown in Figure 2. The data of the MT-HVDC systems are provided in Tables A30-A32.

As it can be observed from Figure 2, the IEEE 39-bus test system is divided into three areas, in which area 1 comprises of three coherent generators (G8, G9, and G10), area 2 comprises of three coherent generators (G1, G2, and G3), and area 3 comprises of four coherent generators (G4, G5, G6, and G7). The main corridors to interconnect those three areas are given as follows:

- $\quad$ Area 1 to Area 2: Lines from bus 1 to bus 39, and from bus 3 to bus 4

- Area 1 to Area 3: Lines from bus 3 to bus 18, and from bus 27 to bus 17

- Area 2 to Area 3: Line from bus 14 to bus 15

The parameters of the converter stations for the PF calculations are as follows:

- Converter Station \#1: $P-Q$ control mode, $P_{g}=-60 \mathrm{MW}$ and $Q_{g}=-20 \mathrm{MVAR}$

- Converter Station \#2: $V_{D C}-V$ control mode, $V_{D C}=1$ p.u. and $V=1$ p.u.

- Converter Station \#3: $P-Q$ control mode, $P_{g}=40 \mathrm{MW}$ and $Q_{g}=40 \mathrm{MVAR}$

- Converter Station \#4: $P-Q$ control mode, $P_{g}=40 \mathrm{MW}$ and $Q_{g}=40 \mathrm{MVAR}$

- Converter Station \#5: $V_{D C}-V$ control mode, $V_{D C}=1$ p.u. and $V=1$ p.u.

- Converter Station \#6: $P-Q$ control mode, $P_{g}=40 \mathrm{MW}$ and $Q_{g}=30 \mathrm{MVAR}$

- Converter Station \#7: $P-Q$ control mode, $P_{g}=40 \mathrm{MW}$ and $Q_{g}=20 \mathrm{MVAR}$

- Converter Station \#8: $P-Q$ control mode, $P_{g}=40 \mathrm{MW}$ and $Q_{g}=40 \mathrm{MVAR}$

From the operation and planning perspectives, the outage of the mentioned lines can cause entirely disconnection of the two areas from each other and accordingly, prevention of power exchange between two areas, operating in islanded mode, and instability of the AC grids. Therefore, there is a need for strengthening the power transmission lines among the mentioned areas. To do so, two four-bus MT-HVDC systems are considered to interconnect the mentioned areas together. Areas 1 and 3 are interconnected with four converter stations (CS1, CS2, CS3, and CS4) and areas 2 and 3 are interconnected with four converter stations (CS5, CS6, CS7, and CS8). Hence, in case of AC lines outage, the power can be transferred via DC links. Tables A33 and A34 show the results of AC PF on the IEEE 39-bus test system without DC grids (Case 7-1).

Tables A35-A38 show the obtained results of the mixed AC/DC PF calculations when MT-HVDC systems are connected to the IEEE 39-bus test system (Case 7-2). In a general view, due to the fact that by interconnecting the MT-HVDC systems to the grid, more power is required, the total generated 
active and reactive power by the generators should increase and decrease, respectively. There is no connection between the MT-HVDC systems and any of the generators. Therefore, only the voltage magnitude and angles are changed. This should be considered that each converter has its own power losses. Therefore, compared to the AC PF, the total power losses on the AC lines in the mixed AC/DC $\mathrm{PF}$ is increased by $0.11 \mathrm{MW}$. There are some slight changes in the total active power generated by the generator \#31. However, the generated reactive power by all the generators is changed. The changes of the PF on the AC lines 1-39, 3-4, 3-18, 4-14, 14-15, 15-16, and 17-27 are significant since they are directly connected to the MT-HVDC systems. To check the changes of the PF on the other lines, the connected lines to the mentioned lines are checked and it is observed that there are some slight changes of the PF on the lines 4-5 and 13-14. Based on the obtained results, the slack converters are capable of transferring power on the DC lines based on the total demand. It is also noticed that compared to the other DC lines, the changes of the PF on the lines 5-6, 5-7, and 5-8 are considerable and the changes of the PF on the DC lines 6-7 and 6-8 are almost zero.

To minimize the power losses, in the next case, it is considered that the converters are equipped with the droop controllers. Table A39 illustrates the detailed information of the droop settings for each converter station. Tables A40-A43 illustrate the results of mixed AC/DC PF considering droop control settings provided in Table A39 (Case 7-3). Analyzing the obtained results shows that the changes of the PF on the AC lines 1-39, 2-3, and 17-27 are almost negligible but the total power losses on the AC lines are decreased. The DC lines 1-4 and 2-3 have the most power losses. In addition, no power is transferred on lines 6-7 and 6-8. Therefore, they can be considered as reserve lines for planning purposes. It is worth mentioning that compared to Case 7-2, the total power losses on the DC lines are decreased.

To analyze the impact of MT-HVDC systems on the PF analysis, AC lines 3-4, 3-18, 4-14, and 14-15 as the main interconnected corridors between each of area are disconnected. Tables A44 and A45 show the results of AC PF without DC grids in the case of disconnecting the AC lines 3-4, 3-18, 4-14, and 14-15 (Case 7-4). The disconnection of the mentioned lines is caused that some of the lines reach their maximum transfer power capabilities. The changes of the PF on the AC lines 4-5, 5-6, 6-11, and 10-11 are drastically changed. However, the changes of the PF on the AC lines 10-13 and 13-14 are deceased. The rest of the lines have either no or very slight changes on the PF.

To improve the reliability of the hybrid AC/DC grids, the MT-HVDC systems are connected to compensate for the disconnections of the AC lines 3-4, 3-18, 4-14, and 14-15. Tables A46-A49 show the obtained results of the mixed AC/DC PF calculations when MT-HVDC systems are connected to the IEEE 39-bus test system considering the disconnections of the AC lines 3-4, 3-18, 4-14, and 14-15 (Case 7-5). Based on the obtained results, the significant changes on the PF are related to the AC lines 1-2, $1-39,10-13,13-14$, and 17-27 (increase) and 2-25, 5-6, 6-11, 10-11, 15-16, 17-18, and 26-27 (decrease). The total power losses on the AC lines are closed to the previous case. In addition, DC lines $5-6,5-7$, and 5-8 are the DC lines with high power losses.

In order to minimize the power losses, it is assumed that the converter stations are equipped with the droop controllers as Table A39. Tables A50-A53 show the obtained results of the mixed AC/DC PF calculations when MT-HVDC systems are connected to the IEEE 39-bus test system considering the droop parameters and disconnections of the AC lines 3-4, 3-18, 4-14, and 14-15 (Case 7-6). The obtained results show that compared to the previous case, only the PF of the AC lines 4-5, 5-6, 15-16, 17-18, and 26-27 are increased and the total power losses both on the AC and DC lines are decreased. 


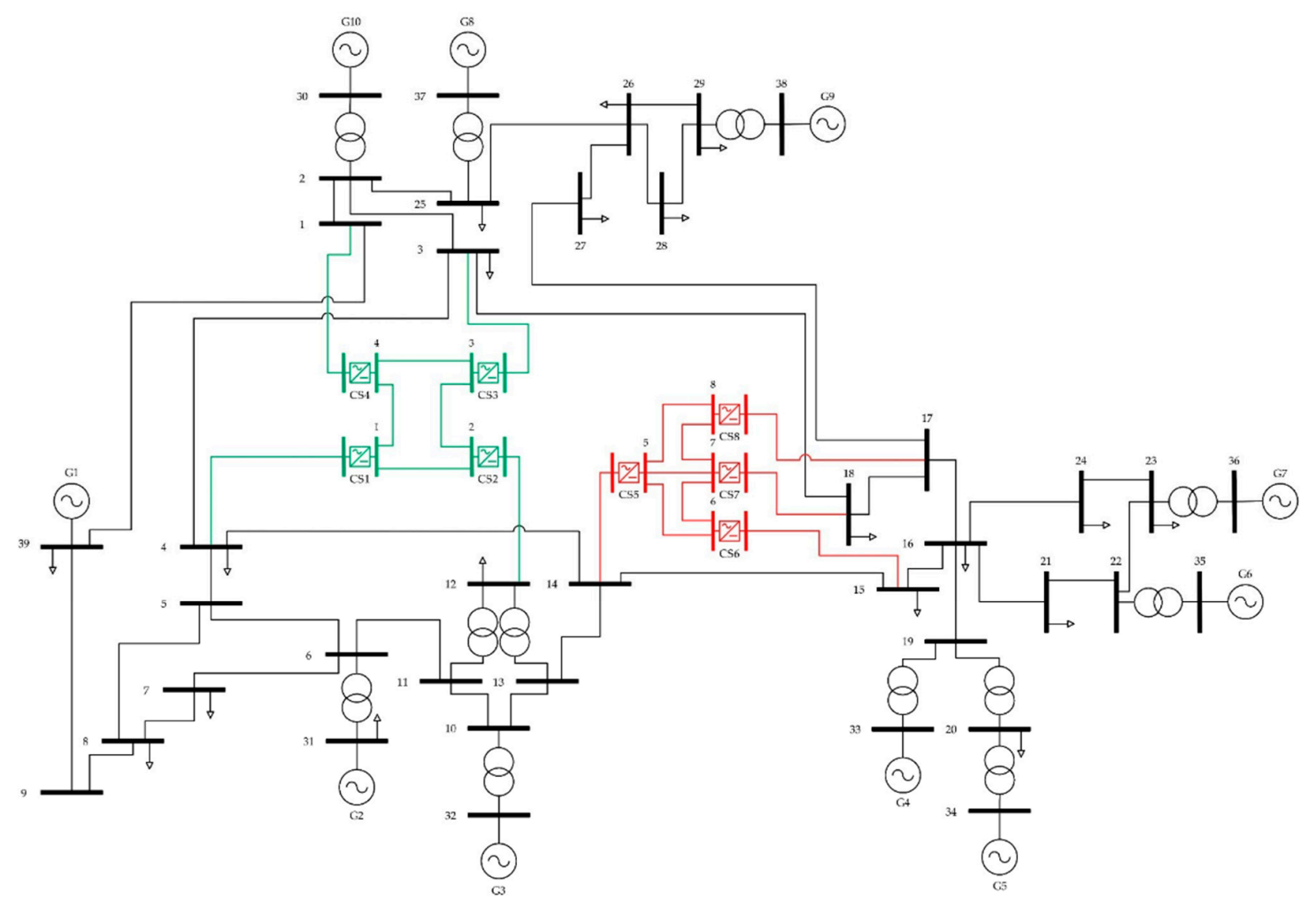

Figure 2. Single-line diagram of the modified IEEE 39-bus test system with two four-bus MT-HVDC systems.

\section{Conclusions}

In this paper, a mixed AC/DC Power Flow (PF) algorithm for the steady-state interaction of the large-scale MT-HVDC systems is investigated. This algorithm is an improved sequential AC/DC PF algorithm, which uses the Newton-Raphson method to solve the DC PF problem. Different operational constraints and control strategies along with contingency analysis in the hybrid AC/DC grids are considered in this study. Fast convergence and high accuracy are the main advantages of the mixed AC/DC PF algorithm. In addition, it is a powerful tool for sensitivity analysis and congestion management in power systems. Various cases are studied in this paper to evaluate the performance of the mixed AC/DC PF algorithm. The obtained results demonstrate the robustness and effectiveness of the mixed AC/DC PF algorithm for power system operation and planning studies.

Author Contributions: F.M. was responsible for methodology, collecting resources, data analysis, writing-original draft preparation, and writing-review and editing. G.-A.N. and M.S. were responsible for the supervision, and writing - review and editing. All authors have read and agreed to the published version of the manuscript.

Funding: M. Saif acknowledges the financial support of Natural Sciences and Engineering Research Council (NSERC) of Canada, as well as National Science Foundation of China under grant number 61873144.

Conflicts of Interest: The authors declare no conflict of interest.

\section{Appendix A}

Note: $P$ and $Q$ are in $M W$ and $M V A R$, respectively. 
Table A1. Results of AC PF calculations in Case 1. AC bus data.

\begin{tabular}{ccccccc}
\hline \multirow{2}{*}{ Bus } & \multicolumn{2}{c}{ Voltage } & \multicolumn{2}{c}{ Generation } & \multicolumn{2}{c}{ Load } \\
\cline { 2 - 7 } & $\mid \boldsymbol{V |}$ (p.u.) & $\boldsymbol{\theta}\left(^{\circ}\right)$ & $\boldsymbol{P}$ & $\boldsymbol{Q}$ & $\boldsymbol{P}$ & $\boldsymbol{Q}$ \\
\hline 1 & 1.060 & 0.000 & 131.12 & 90.82 & - & - \\
2 & 1.000 & -2.061 & 40.00 & -61.59 & 20.00 & 10.00 \\
3 & 0.987 & -4.637 & - & - & 45.00 & 15.00 \\
4 & 0.984 & -4.957 & - & - & 40.00 & 5.00 \\
5 & 0.972 & -5.765 & - & - & 60.00 & 10.00 \\
& Total: & & 171.12 & 29.23 & 165.00 & 40.00 \\
\hline
\end{tabular}

Table A2. Results of AC PF calculations in Case 1. AC branch data.

\begin{tabular}{ccccccccc}
\hline \multirow{2}{*}{ Branch } & \multicolumn{9}{c}{ From Bus Injection } & \multicolumn{2}{c}{ To Bus Injection } & \multicolumn{2}{c}{ Power Losses } \\
\cline { 2 - 9 } & From & To & $\boldsymbol{P}$ & $\boldsymbol{Q}$ & $\boldsymbol{P}$ & $\boldsymbol{Q}$ & $\boldsymbol{P}$ & $\boldsymbol{Q}$ \\
\hline 1 & 1 & 2 & 89.33 & 74.00 & -86.85 & -72.91 & 2.486 & 7.46 \\
2 & 1 & 3 & 41.79 & 16.82 & -40.27 & -17.51 & 1.518 & 4.55 \\
3 & 2 & 3 & 24.47 & -2.52 & -24.11 & -0.35 & 0.360 & 1.08 \\
4 & 2 & 4 & 27.71 & -1.72 & -27.25 & -0.83 & 0.461 & 1.38 \\
5 & 2 & 5 & 54.66 & 5.56 & -53.44 & -4.83 & 1.215 & 3.65 \\
6 & 3 & 4 & 19.39 & 2.86 & -19.35 & -4.69 & 0.040 & 0.12 \\
7 & 4 & 5 & 6.60 & 0.52 & -6.56 & -5.17 & 0.043 & 0.13 \\
& & & & & & Total: & 6.123 & 18.37 \\
\hline
\end{tabular}

Table A3. Results of the mixed AC/DC PF calculations in Case 2. AC bus data.

\begin{tabular}{ccccccc}
\hline \multirow{2}{*}{ Bus } & \multicolumn{2}{c}{ Voltage } & \multicolumn{2}{c}{ Generation } & \multicolumn{2}{c}{ Load } \\
\cline { 2 - 7 } & $|\boldsymbol{V}|$ (p.u.) & $\boldsymbol{\theta}\left(^{\circ}\right)$ & $\boldsymbol{P}$ & $\boldsymbol{Q}$ & $\boldsymbol{P}$ & $\boldsymbol{Q}$ \\
\hline 1 & 1.060 & 0.000 & 133.64 & 84.32 & - & - \\
2 & 1.000 & -2.383 & 40.00 & -32.84 & 20.00 & 10.00 \\
3 & 1.000 & -3.895 & - & - & 45.00 & 15.00 \\
4 & 0.996 & -4.262 & - & - & 40.00 & 5.00 \\
5 & 0.991 & -4.149 & - & - & 60.00 & 10.00 \\
& Total: & & 173.64 & 51.48 & 165.00 & 40.00 \\
\hline
\end{tabular}

Table A4. Results of the mixed AC/DC PF calculations in Case 2. AC branch data.

\begin{tabular}{ccccccccc}
\hline \multirow{2}{*}{ Branch } & \multicolumn{4}{c}{ From Bus Injection } & \multicolumn{2}{c}{ To Bus Injection } & \multicolumn{2}{c}{ Power Losses } \\
\cline { 2 - 9 } & From & To & $\boldsymbol{P}$ & $\boldsymbol{Q}$ & $\boldsymbol{P}$ & $\boldsymbol{Q}$ & $\boldsymbol{P}$ & $\boldsymbol{Q}$ \\
\hline 1 & 1 & 2 & 98.38 & 71.37 & -95.66 & -69.59 & 2.717 & 8.15 \\
2 & 1 & 3 & 35.26 & 12.96 & -34.20 & -15.08 & 1.062 & 3.19 \\
3 & 2 & 3 & 13.25 & -6.22 & -13.14 & 2.57 & 0.116 & 0.35 \\
4 & 2 & 4 & 17.08 & -5.18 & -16.89 & 1.74 & 0.181 & 0.54 \\
5 & 2 & 5 & 25.33 & -1.85 & -25.07 & -0.35 & 0.257 & 0.77 \\
6 & 3 & 4 & 23.09 & 4.64 & -23.04 & -6.47 & 0.057 & 0.17 \\
7 & 4 & 5 & -0.07 & -0.27 & 0.07 & -4.65 & 0.004 & 0.01 \\
& & & & & & Total: & 4.394 & 13.18 \\
\hline
\end{tabular}

Table A5. Results of the mixed AC/DC PF calculations in Case 2. DC bus data.

\begin{tabular}{cccc}
\hline Bus DC & Bus AC & Voltage Magnitude (p.u.) & Active Power (MW) \\
\hline 1 & 2 & 1.008 & -58.627 \\
2 & 3 & 1.000 & 21.901 \\
3 & 5 & 0.998 & 36.186 \\
\hline
\end{tabular}


Table A6. Results of the mixed AC/DC PF calculations in Case 2. Converter station data.

\begin{tabular}{|c|c|c|c|c|c|c|c|c|}
\hline \multirow{2}{*}{ Bus DC } & \multicolumn{3}{|c|}{ Bus Injection } & \multicolumn{3}{|c|}{ Converter Voltage } & \multicolumn{2}{|c|}{ Total Loss } \\
\hline & \multicolumn{2}{|c|}{$P$} & $Q$ & \multicolumn{2}{|c|}{$|V|$ (p.u.) } & $\theta\left({ }^{\circ}\right)$ & \multicolumn{2}{|c|}{$P$} \\
\hline 1 & \multicolumn{2}{|c|}{-60.00} & -40.00 & \multicolumn{2}{|c|}{0.890} & -13.017 & \multicolumn{2}{|c|}{1.37} \\
\hline 2 & \multicolumn{2}{|c|}{20.76} & 7.14 & \multicolumn{2}{|c|}{1.007} & -0.655 & \multicolumn{2}{|c|}{1.14} \\
\hline 3 & \multirow{2}{*}{\multicolumn{2}{|c|}{35.00}} & 5.00 & \multirow{2}{*}{\multicolumn{2}{|c|}{0.995}} & 1.442 & \multirow{2}{*}{\multicolumn{2}{|c|}{$\begin{array}{l}1.19 \\
3.70\end{array}$}} \\
\hline & & & & & & Total: & & \\
\hline \multirow{2}{*}{ Bus DC } & \multicolumn{2}{|c|}{ Converter Power } & Filter & \multicolumn{2}{|c|}{ Transformer Loss } & \multicolumn{2}{|c|}{ Reactor Loss } & Converter Loss \\
\hline & $P$ & $Q$ & $Q$ & $P$ & $Q$ & $P$ & $Q$ & $P$ \\
\hline 1 & -59.22 & -32.63 & -8.12 & 0.08 & 5.83 & 0.01 & 9.66 & 1.29 \\
\hline 2 & 20.76 & -0.65 & -9.02 & 0.01 & 0.54 & 0.00 & 0.70 & 1.14 \\
\hline \multirow[t]{2}{*}{3} & \multirow[t]{2}{*}{35.02} & \multirow[t]{2}{*}{-0.37} & -8.83 & 0.02 & 1.43 & 0.00 & 2.03 & 1.17 \\
\hline & & & Total: & 0.11 & 7.80 & 0.01 & 12.39 & 3.60 \\
\hline \multirow[t]{2}{*}{ Bus DC } & \multicolumn{2}{|c|}{ Grid Power } & \multicolumn{2}{|c|}{ Trans. Filter Power } & \multirow{2}{*}{$\begin{array}{c}\text { Filter } \\
Q\end{array}$} & \multirow{2}{*}{$\begin{array}{c}\begin{array}{c}\text { Conv. Filter } \\
\text { Power }\end{array} \\
Q\end{array}$} & \multicolumn{2}{|c|}{ Conv. Power } \\
\hline & $P$ & $Q$ & $P$ & $Q$ & & & $P$ & $Q$ \\
\hline 1 & -60.00 & -40.00 & -59.92 & -34.17 & -8.12 & -42.29 & -59.92 & -32.63 \\
\hline 2 & 20.76 & 7.14 & 20.76 & 7.68 & -9.02 & -1.35 & 20.76 & -0.65 \\
\hline 3 & 35.00 & 5.00 & 35.02 & 6.43 & -8.83 & -2.40 & 35.02 & -0.37 \\
\hline
\end{tabular}

Table A7. Results of the mixed AC/DC PF calculations in Case 2. DC branch data.

\begin{tabular}{|c|c|c|c|c|c|}
\hline \multirow{2}{*}{ Branch } & \multicolumn{3}{|c|}{ From Bus } & \multirow{2}{*}{$\begin{array}{c}\text { To Bus } \\
P\end{array}$} & \multirow{2}{*}{$\begin{array}{c}\text { Power Losses } \\
P\end{array}$} \\
\hline & From & To & $P$ & & \\
\hline 1 & 1 & 2 & 30.66 & -30.42 & 0.24 \\
\hline 2 & 2 & 3 & 8.52 & -8.50 & 0.02 \\
\hline \multirow[t]{2}{*}{3} & 1 & 3 & 27.96 & -27.68 & 0.28 \\
\hline & & & & Total: & 0.54 \\
\hline
\end{tabular}

Table A8. Results of the mixed AC/DC PF calculations in Case 3. AC bus data.

\begin{tabular}{ccccc}
\hline \multirow{2}{*}{ Bus } & \multicolumn{2}{c}{ Generation } & \multicolumn{2}{c}{ Load } \\
\cline { 2 - 5 } & $\boldsymbol{P}$ & $\boldsymbol{Q}$ & $\boldsymbol{P}$ & $\boldsymbol{Q}$ \\
\hline 1 & 133.93 & 84.93 & - & - \\
2 & 40.00 & -90.48 & 20.00 & 10.00 \\
3 & - & - & 45.00 & 15.00 \\
4 & - & - & 40.00 & 5.00 \\
5 & - & - & 60.00 & 10.00 \\
Total: & 173.93 & -5.55 & 165.00 & 40.00 \\
\hline
\end{tabular}

Table A9. Results of the mixed AC/DC PF calculations in Case 3. AC branch data.

\begin{tabular}{ccccccccc}
\hline \multirow{2}{*}{ Branch } & \multicolumn{9}{c}{ From Bus Injection } & \multicolumn{2}{c}{ To Bus Injection } & \multicolumn{2}{c}{ Power Losses } \\
\cline { 2 - 9 } & From & To & $\boldsymbol{P}$ & $\boldsymbol{Q}$ & $\boldsymbol{P}$ & $\boldsymbol{Q}$ & $\boldsymbol{P}$ & $\boldsymbol{Q}$ \\
\hline 1 & 1 & 2 & 84.95 & 75.29 & -82.56 & -74.50 & 2.386 & 7.16 \\
2 & 1 & 3 & 48.98 & 9.64 & -47.17 & -9.50 & 1.819 & 5.46 \\
3 & 2 & 3 & 34.58 & -12.23 & -33.80 & 10.57 & 0.780 & 2.34 \\
4 & 2 & 4 & 34.13 & -9.79 & -33.40 & 8.01 & 0.735 & 2.21 \\
5 & 2 & 5 & 33.85 & -3.96 & -33.39 & 2.38 & 0.461 & 1.38 \\
6 & 3 & 4 & -1.69 & 13.76 & 1.71 & -15.69 & 0.022 & 0.07 \\
7 & 4 & 5 & -8.31 & 2.68 & 8.39 & -7.38 & 0.077 & 0.23 \\
& & & & & & Total: & 6.283 & 18.85 \\
\hline
\end{tabular}


Table A10. Results of the mixed AC/DC PF calculations in Case 3. DC bus data.

\begin{tabular}{cccc}
\hline \multirow{2}{*}{ Bus DC } & \multicolumn{2}{c}{ Bus Injection } & Total Loss \\
\cline { 2 - 4 } & $\boldsymbol{P}$ & $\boldsymbol{Q}$ & $\boldsymbol{P}$ \\
\hline 1 & 0.00 & 0.00 & 0.00 \\
2 & -37.65 & 29.84 & 1.22 \\
3 & 35.00 & 5.00 & 1.19 \\
& & Total: & 2.41 \\
\hline
\end{tabular}

Table A11. Results of the mixed AC/DC PF calculations in Case 3. DC branch data.

\begin{tabular}{|c|c|c|c|c|c|}
\hline \multirow{2}{*}{ Branch } & \multicolumn{3}{|r|}{ From Bus } & \multirow{2}{*}{$\begin{array}{c}\text { To Bus } \\
P\end{array}$} & \multirow{2}{*}{$\begin{array}{c}\text { Power Losses } \\
P\end{array}$} \\
\hline & From & To & $P$ & & \\
\hline 1 & 1 & 2 & -10.67 & 10.70 & 0.03 \\
\hline 2 & 2 & 3 & 25.73 & -25.55 & 0.17 \\
\hline \multirow[t]{2}{*}{3} & 1 & 3 & 10.67 & -10.63 & 0.04 \\
\hline & & & & Total: & 0.24 \\
\hline
\end{tabular}

Table A12. Droop control settings for each converter station.

\begin{tabular}{cccc}
\hline Converter Station & Droop Parameter & $P_{D C}^{*}(M W)$ & $V_{D C}^{*}(p . u)$. \\
\hline 1 & 0.005 & -58.6274 & 1.0079 \\
2 & 0.007 & 21.9013 & 1.0000 \\
3 & 0.005 & 36.1856 & 0.9778 \\
\hline${ }^{*} P_{D C}^{*}$ and $V_{D C}^{*}$ are the reference power and reference DC voltage, respectively.
\end{tabular}

Table A13. Results of the mixed AC/DC PF calculations in Case 4. AC bus data.

\begin{tabular}{ccccc}
\hline \multirow{2}{*}{ Bus } & \multicolumn{2}{c}{ Generation } & \multicolumn{2}{c}{ Load } \\
\cline { 2 - 5 } & $\boldsymbol{P}$ & $\boldsymbol{Q}$ & $\boldsymbol{P}$ & $\boldsymbol{Q}$ \\
\hline 1 & 133.39 & 84.72 & - & - \\
2 & 40.00 & -81.05 & 20.00 & 10.00 \\
3 & - & - & 45.00 & 15.00 \\
4 & - & - & 40.00 & 5.00 \\
5 & - & - & 60.00 & 10.00 \\
Total: & 173.39 & 0.67 & 165.00 & 40.00 \\
\hline
\end{tabular}

Table A14. Results of the mixed AC/DC PF calculations in Case 4. AC branch data.

\begin{tabular}{ccccccccc}
\hline \multirow{2}{*}{ Branch } & \multicolumn{4}{c}{ From Bus Injection } & \multicolumn{2}{c}{ To Bus Injection } & \multicolumn{2}{c}{ Power Losses } \\
\cline { 2 - 9 } & From & To & $\boldsymbol{P}$ & $\boldsymbol{Q}$ & $\boldsymbol{P}$ & $\boldsymbol{Q}$ & $\boldsymbol{P}$ & $\boldsymbol{Q}$ \\
\hline 1 & 1 & 2 & 90.34 & 73.70 & -87.83 & -72.54 & 2.510 & 7.53 \\
2 & 1 & 3 & 43.05 & 11.02 & -41.59 & -11.96 & 1.456 & 4.37 \\
3 & 2 & 3 & 25.47 & -9.78 & -25.05 & 7.06 & 0.426 & 1.28 \\
4 & 2 & 4 & 28.39 & -7.76 & -27.88 & 5.29 & 0.503 & 1.51 \\
5 & 2 & 5 & 53.97 & -0.97 & -52.81 & 1.52 & 1.165 & 3.50 \\
6 & 3 & 4 & 18.07 & 10.21 & -18.03 & -12.07 & 0.045 & 0.14 \\
7 & 4 & 5 & 5.91 & 1.77 & -5.87 & -6.52 & 0.043 & 0.13 \\
& & & & & & Total: & 6.148 & 18.46 \\
\hline
\end{tabular}


Table A15. Results of the mixed AC/DC PF calculations in Case 4. DC bus data.

\begin{tabular}{cccc}
\hline \multirow{2}{*}{ Bus DC } & \multicolumn{2}{c}{ Bus Injection } & Total Loss \\
\cline { 2 - 4 } & $\boldsymbol{P}$ & $\boldsymbol{Q}$ & $\boldsymbol{P}$ \\
\hline 1 & 0.00 & 0.00 & 0.00 \\
2 & -3.57 & 20.31 & 1.13 \\
3 & 1.33 & 5.00 & 1.11 \\
& Total: & & 2.24 \\
\hline
\end{tabular}

Table A16. Results of the mixed AC/DC PF calculations in Case 4. DC branch data.

\begin{tabular}{|c|c|c|c|c|c|}
\hline \multirow{2}{*}{ Branch } & \multicolumn{3}{|r|}{ From Bus } & \multirow{2}{*}{$\begin{array}{c}\text { To Bus } \\
P\end{array}$} & \multirow{2}{*}{$\begin{array}{c}\text { Power Losses } \\
P\end{array}$} \\
\hline & From & To & $P$ & & \\
\hline 1 & 1 & 2 & -0.72 & 0.72 & 0.00 \\
\hline 2 & 2 & 3 & 1.72 & -1.72 & 0.00 \\
\hline \multirow[t]{2}{*}{3} & 1 & 3 & 0.72 & -0.72 & 0.00 \\
\hline & & & & Total: & 0.00 \\
\hline
\end{tabular}

Table A17. Changes in the droop parameters of each converter station.

\begin{tabular}{cc}
\hline Converter Station & Droop Parameter \\
\hline 1 & 0.0010 \\
2 & 0.0014 \\
3 & 0.0010 \\
\hline
\end{tabular}

Table A18. Results of the mixed AC/DC PF calculations in Case 5. AC bus data.

\begin{tabular}{ccccc}
\hline \multirow{2}{*}{ Bus } & \multicolumn{2}{c}{ Generation } & \multicolumn{2}{c}{ Load } \\
\cline { 2 - 5 } & $\boldsymbol{P}$ & $\boldsymbol{Q}$ & $\boldsymbol{P}$ & $\boldsymbol{Q}$ \\
\hline 1 & 133.38 & 84.72 & - & - \\
2 & 40.00 & -81.22 & 20.00 & 10.00 \\
3 & - & - & 45.00 & 15.00 \\
4 & - & - & 40.00 & 5.00 \\
5 & - & - & 60.00 & 10.00 \\
Total: & 173.38 & 3.50 & 165.00 & 40.00 \\
\hline
\end{tabular}

Table A19. Results of the mixed AC/DC PF calculations in Case 5. AC branch data.

\begin{tabular}{ccccccccc}
\hline \multirow{2}{*}{ Branch } & \multicolumn{4}{c}{ From Bus Injection } & \multicolumn{2}{c}{ To Bus Injection } & \multicolumn{2}{c}{ Power Losses } \\
\cline { 2 - 9 } & From & To & $\boldsymbol{P}$ & $\boldsymbol{Q}$ & $\boldsymbol{P}$ & $\boldsymbol{Q}$ & $\boldsymbol{P}$ & $\boldsymbol{Q}$ \\
\hline 1 & 1 & 2 & 90.24 & 73.73 & -87.74 & -72.58 & 2.508 & 7.52 \\
2 & 1 & 3 & 43.14 & 11.00 & -41.68 & -11.92 & 1.461 & 4.38 \\
3 & 2 & 3 & 25.62 & -9.82 & -25.19 & 7.11 & 0.430 & 1.29 \\
4 & 2 & 4 & 28.48 & -7.80 & -27.97 & 5.34 & 0.507 & 1.52 \\
5 & 2 & 5 & 53.64 & -1.02 & -52.49 & 1.54 & 1.151 & 3.45 \\
6 & 3 & 4 & 17.75 & 10.26 & -17.71 & -12.12 & 0.044 & 0.13 \\
7 & 4 & 5 & 5.68 & 1.78 & -5.64 & -6.54 & 0.041 & 0.12 \\
& & & & & & Total: & 6.142 & 18.41 \\
\hline
\end{tabular}


Table A20. Results of the mixed AC/DC PF calculations in Case 5. DC bus data.

\begin{tabular}{cccc}
\hline \multirow{2}{*}{ Bus DC } & \multicolumn{2}{c}{ Bus Injection } & Total Loss \\
\cline { 2 - 4 } & $\boldsymbol{P}$ & $\boldsymbol{Q}$ & $\boldsymbol{P}$ \\
\hline 1 & 0.00 & 0.00 & 0.00 \\
2 & -4.11 & 20.45 & 1.13 \\
3 & 1.87 & 5.00 & 1.11 \\
& Total: & & 2.24 \\
\hline
\end{tabular}

Table A21. Results of the mixed AC/DC PF calculations in Case 5. DC branch data.

\begin{tabular}{|c|c|c|c|c|c|}
\hline \multirow{2}{*}{ Branch } & \multicolumn{3}{|r|}{ From Bus } & \multirow{2}{*}{$\begin{array}{c}\text { To Bus } \\
P\end{array}$} & \multirow{2}{*}{$\begin{array}{c}\text { Power Losses } \\
P\end{array}$} \\
\hline & From & To & $P$ & & \\
\hline 1 & 1 & 2 & -0.88 & 0.88 & 0.00 \\
\hline 2 & 2 & 3 & 2.11 & -2.11 & 0.00 \\
\hline \multirow[t]{2}{*}{3} & 1 & 3 & 0.88 & -0.88 & 0.00 \\
\hline & & & & Total: & 0.00 \\
\hline
\end{tabular}

Table A22. Results of the mixed AC/DC PF calculations in Case 6-1. AC bus data.

\begin{tabular}{ccccc}
\hline \multirow{2}{*}{ Bus } & \multicolumn{2}{c}{ Generation } & \multicolumn{2}{c}{ Load } \\
\cline { 2 - 5 } & $\boldsymbol{P}$ & $\boldsymbol{Q}$ & $\boldsymbol{P}$ & $\boldsymbol{Q}$ \\
\hline 1 & 133.38 & 84.72 & - & - \\
2 & 40.00 & -81.22 & 20.00 & 10.00 \\
3 & - & - & 45.00 & 15.00 \\
4 & - & - & 40.00 & 5.00 \\
5 & - & - & 60.00 & 10.00 \\
Total: & 173.38 & 3.50 & 165.00 & 40.00 \\
\hline
\end{tabular}

Table A23. Results of the mixed AC/DC PF calculations in Case 6-1. AC branch data.

\begin{tabular}{ccccccccc}
\hline \multirow{2}{*}{ Branch } & \multicolumn{9}{c}{ From Bus Injection } & \multicolumn{2}{c}{ To Bus Injection } & \multicolumn{2}{c}{ Power Losses } \\
\cline { 2 - 9 } & From & To & $\boldsymbol{P}$ & $\boldsymbol{Q}$ & $\boldsymbol{P}$ & $\boldsymbol{Q}$ & $\boldsymbol{P}$ & $\boldsymbol{Q}$ \\
\hline 1 & 1 & 2 & 90.24 & 73.73 & -87.74 & -72.58 & 2.508 & 7.52 \\
2 & 1 & 3 & 43.14 & 11.00 & -41.68 & -11.92 & 1.461 & 4.38 \\
3 & 2 & 3 & 25.62 & -9.82 & -25.19 & 7.11 & 0.430 & 1.29 \\
4 & 2 & 4 & 28.48 & -7.80 & -27.97 & 5.34 & 0.507 & 1.52 \\
5 & 2 & 5 & 53.64 & -1.02 & -52.49 & 1.54 & 1.151 & 3.45 \\
6 & 3 & 4 & 17.75 & 10.26 & -17.71 & -12.12 & 0.044 & 0.13 \\
7 & 4 & 5 & 5.68 & 1.78 & -5.64 & -6.54 & 0.041 & 0.12 \\
& & & & & & Total: & 6.142 & 18.41 \\
\hline
\end{tabular}

Table A24. Results of the mixed AC/DC PF calculations in Case 6-1. DC bus data.

\begin{tabular}{cccc}
\hline \multirow{2}{*}{ Bus DC } & \multicolumn{2}{c}{ Bus Injection } & Total Loss \\
\cline { 2 - 4 } & $\boldsymbol{P}$ & $\boldsymbol{Q}$ & $\boldsymbol{P}$ \\
\hline 1 & 0.00 & 0.00 & 0.00 \\
2 & -4.11 & 20.45 & 1.13 \\
3 & 1.87 & 5.00 & 1.11 \\
& Total: & & 2.24 \\
\hline
\end{tabular}


Table A25. Results of the mixed AC/DC PF calculations in Case 6-1. DC branch data.

\begin{tabular}{|c|c|c|c|c|c|}
\hline \multirow{2}{*}{ Branch } & \multicolumn{3}{|r|}{ From Bus } & \multirow{2}{*}{$\begin{array}{c}\text { To Bus } \\
P\end{array}$} & \multirow{2}{*}{$\begin{array}{c}\text { Power Losses } \\
P\end{array}$} \\
\hline & From & To & $P$ & & \\
\hline 1 & 1 & 2 & -0.88 & 0.88 & 0.00 \\
\hline 2 & 2 & 3 & 2.11 & -2.11 & 0.00 \\
\hline \multirow[t]{2}{*}{3} & 1 & 3 & 0.88 & -0.88 & 0.00 \\
\hline & & & & Total: & 0.00 \\
\hline
\end{tabular}

Table A26. Results of the mixed AC/DC PF calculations in Case 6-2. AC bus data.

\begin{tabular}{ccccc}
\hline \multirow{2}{*}{ Bus } & \multicolumn{2}{c}{ Generation } & \multicolumn{2}{c}{ Load } \\
\cline { 2 - 5 } & $\boldsymbol{P}$ & $\boldsymbol{Q}$ & $\boldsymbol{P}$ & $\boldsymbol{Q}$ \\
\hline 1 & 135.63 & 83.69 & - & - \\
2 & 40.00 & -61.38 & 20.00 & 10.00 \\
3 & - & - & 45.00 & 15.00 \\
4 & - & - & 40.00 & 5.00 \\
5 & - & - & 60.00 & 10.00 \\
Total: & 175.63 & 22.31 & 165.00 & 40.00 \\
\hline
\end{tabular}

Table A27. Results of the mixed AC/DC PF calculations in Case 6-2. AC branch data.

\begin{tabular}{ccccccccc}
\hline \multirow{2}{*}{ Branch } & \multicolumn{9}{c}{ From Bus Injection } & \multicolumn{2}{c}{ To Bus Injection } & \multicolumn{2}{c}{ Power Losses } \\
\cline { 2 - 9 } & From & To & $\boldsymbol{P}$ & $\boldsymbol{Q}$ & $\boldsymbol{P}$ & $\boldsymbol{Q}$ & $\boldsymbol{P}$ & $\boldsymbol{Q}$ \\
\hline 1 & 1 & 2 & 113.59 & 67.07 & -110.41 & -63.90 & 3.180 & 9.54 \\
2 & 1 & 3 & 22.04 & 16.62 & -21.42 & -20.08 & 0.615 & 1.84 \\
3 & 2 & 3 & -7.87 & 0.69 & 7.91 & -4.57 & 0.042 & 0.12 \\
4 & 2 & 4 & 0.18 & 0.16 & -0.18 & -4.13 & 0.003 & 0.01 \\
5 & 2 & 5 & 16.89 & 0.63 & -16.78 & -3.25 & 0.116 & 0.35 \\
6 & 3 & 4 & 48.34 & -3.45 & -48.10 & 2.16 & 0.234 & 0.70 \\
7 & 4 & 5 & 8.28 & -3.02 & -8.22 & -1.75 & 0.056 & 0.17 \\
& & & & & & Total: & 4.246 & 12.73 \\
\hline
\end{tabular}

Table A28. Results of the mixed AC/DC PF calculations in Case 6-2. DC bus data.

\begin{tabular}{cccc}
\hline \multirow{2}{*}{ Bus DC } & \multicolumn{2}{c}{ Bus Injection } & Total Loss \\
\cline { 2 - 4 } & $\boldsymbol{P}$ & $\boldsymbol{Q}$ & $\boldsymbol{P}$ \\
\hline 1 & -121.21 & 8.96 & 1.69 \\
2 & 79.82 & -13.10 & 1.38 \\
3 & 35.00 & 5.00 & 1.19 \\
& Total: & & 4.26 \\
\hline
\end{tabular}

Table A29. Results of the mixed AC/DC PF calculations in Case 6-2. DC branch data.

\begin{tabular}{|c|c|c|c|c|c|}
\hline \multirow{2}{*}{ Branch } & \multicolumn{3}{|c|}{ From Bus } & \multirow{2}{*}{$\begin{array}{c}\text { To Bus } \\
P\end{array}$} & \multirow{2}{*}{$\begin{array}{c}\text { Power Losses } \\
P\end{array}$} \\
\hline & From & To & $P$ & & \\
\hline 1 & 1 & 2 & 73.60 & -72.24 & 1.36 \\
\hline 2 & 2 & 3 & -8.97 & 8.99 & 0.02 \\
\hline \multirow[t]{2}{*}{3} & 1 & 3 & 45.92 & -45.18 & 0.74 \\
\hline & & & & Total: & 2.12 \\
\hline
\end{tabular}


Table A30. Parameters of the converter stations in per-unit.

\begin{tabular}{ccccccc}
\hline Converter Station & $\boldsymbol{S}_{\boldsymbol{N}}(\mathbf{M V A})$ & $\boldsymbol{R}_{\boldsymbol{T R}}$ & $\boldsymbol{X}_{\boldsymbol{T R}}$ & $\boldsymbol{B}_{\boldsymbol{F}}$ & $\boldsymbol{R}_{\boldsymbol{T R}}$ & $\boldsymbol{X}_{\boldsymbol{T R}}$ \\
\hline 1 & 100 & 0.0015 & 0.1121 & 0.0887 & 0.0001 & 0.1643 \\
2 & 100 & 0.0015 & 0.1121 & 0.0887 & 0.0001 & 0.1643 \\
3 & 100 & 0.0015 & 0.1121 & 0.0887 & 0.0001 & 0.1643 \\
4 & 100 & 0.0015 & 0.1121 & 0.0887 & 0.0001 & 0.1643 \\
5 & 100 & 0.0015 & 0.1121 & 0.0887 & 0.0001 & 0.1643 \\
6 & 100 & 0.0015 & 0.1121 & 0.0887 & 0.0001 & 0.1643 \\
7 & 100 & 0.0015 & 0.1121 & 0.0887 & 0.0001 & 0.1643 \\
\hline
\end{tabular}

Table A31. Power losses coefficients of the converter stations.

\begin{tabular}{ccccc}
\hline Converter Station & $\boldsymbol{a}$ & $\boldsymbol{b}$ & $\boldsymbol{c}_{\text {rec }}$ & $\boldsymbol{c}_{\boldsymbol{i n v}}$ \\
\hline 1 & 1.103 & 0.887 & 2.885 & 4.371 \\
2 & 1.103 & 0.887 & 2.885 & 4.371 \\
3 & 1.103 & 0.887 & 2.885 & 4.371 \\
4 & 1.103 & 0.887 & 2.885 & 4.371 \\
5 & 1.103 & 0.887 & 2.885 & 4.371 \\
6 & 1.103 & 0.887 & 2.885 & 4.371 \\
7 & 1.103 & 0.887 & 2.885 & 4.371 \\
\hline
\end{tabular}

Table A32. Parameters of the DC lines.

\begin{tabular}{ccccc}
\hline From & To & $\boldsymbol{R}_{\boldsymbol{D C}}$ (p.u.) & $\boldsymbol{V}_{\boldsymbol{D C}}(\mathbf{k V )}$ & $\boldsymbol{P}_{\boldsymbol{D C}}(\mathbf{M W})$ \\
\hline 1 & 2 & 0.0520 & 345 & 100 \\
1 & 4 & 0.0520 & 345 & 100 \\
2 & 3 & 0.0520 & 345 & 100 \\
3 & 4 & 0.0520 & 345 & 100 \\
5 & 6 & 0.0730 & 345 & 100 \\
5 & 7 & 0.0730 & 345 & 100 \\
5 & 8 & 0.0730 & 345 & 100 \\
6 & 7 & 0.0730 & 345 & 100 \\
6 & 8 & 0.0730 & 345 & 100 \\
\hline
\end{tabular}


Table A33. Results of AC PF calculations in Case 7-1. AC bus data.

\begin{tabular}{|c|c|c|c|c|c|c|}
\hline \multirow{2}{*}{ Bus } & \multicolumn{2}{|c|}{ Voltage } & \multicolumn{2}{|c|}{ Generation } & \multicolumn{2}{|c|}{ Load } \\
\hline & $|V|$ (p.u.) & $\theta\left({ }^{\circ}\right)$ & $P$ & $Q$ & $P$ & $Q$ \\
\hline 1 & 1.039 & -13.537 & - & - & 97.60 & 44.20 \\
\hline 2 & 1.048 & -9.785 & - & - & - & - \\
\hline 3 & 1.031 & -12.276 & - & - & 322.00 & 2.40 \\
\hline 4 & 1.004 & -12.627 & - & - & 500.00 & 184.00 \\
\hline 5 & 1.006 & -11.192 & - & - & - & - \\
\hline 6 & 1.008 & -10.408 & - & - & - & - \\
\hline 7 & 0.998 & -12.756 & - & - & 233.80 & 84.00 \\
\hline 8 & 0.998 & -13.336 & - & - & 522.00 & 176.60 \\
\hline 9 & 1.038 & -14.178 & - & - & 6.50 & -66.60 \\
\hline 10 & 1.018 & -8.171 & - & - & - & - \\
\hline 11 & 1.013 & -8.937 & - & - & - & - \\
\hline 12 & 1.001 & -8.999 & - & - & 8.53 & 88.00 \\
\hline 13 & 1.015 & -8.930 & - & - & - & - \\
\hline 14 & 1.012 & -10.715 & - & - & - & - \\
\hline 15 & 1.016 & -11.345 & - & - & 320.00 & 153.00 \\
\hline 16 & 1.033 & -10.033 & - & - & 329.00 & 32.30 \\
\hline 17 & 1.034 & -11.116 & - & - & - & - \\
\hline 18 & 1.032 & -11.986 & - & - & 158.00 & 30.00 \\
\hline 19 & 1.050 & -5.410 & - & - & - & - \\
\hline 20 & 0.991 & -6.821 & - & - & 680.00 & 103.00 \\
\hline 21 & 1.032 & -7.629 & - & - & 274.00 & 115.00 \\
\hline 22 & 1.050 & -3.183 & - & - & - & - \\
\hline 23 & 1.045 & -3.381 & - & - & 247.50 & 84.60 \\
\hline 24 & 1.038 & -9.914 & - & - & 308.60 & -92.20 \\
\hline 25 & 1.058 & -8.369 & - & - & 224.00 & 47.20 \\
\hline 26 & 1.053 & -9.439 & - & - & 139.00 & 17.00 \\
\hline 27 & 1.038 & -11.362 & - & - & 281.00 & 75.50 \\
\hline 28 & 1.050 & -5.928 & - & - & 206.00 & 27.60 \\
\hline 29 & 1.050 & -3.170 & - & - & 283.50 & 26.90 \\
\hline 30 & 1.050 & -7.370 & 250.00 & 161.76 & - & - \\
\hline 31 & 0.982 & 0.000 & 677.87 & 221.57 & 9.20 & 4.60 \\
\hline 32 & 0.984 & -0.188 & 650.00 & 206.96 & - & - \\
\hline 33 & 0.997 & -0.196 & 632.00 & 108.29 & - & - \\
\hline 34 & 1.012 & -1.631 & 508.00 & 166.69 & - & - \\
\hline 35 & 1.049 & 1.777 & 650.00 & 210.66 & - & - \\
\hline 36 & 1.064 & 4.468 & 560.00 & 100.16 & - & - \\
\hline 37 & 1.028 & -1.583 & 540.00 & -1.37 & - & - \\
\hline 38 & 1.027 & 3.893 & 830.00 & 21.73 & - & - \\
\hline \multirow[t]{2}{*}{39} & 1.030 & -14.535 & 1000.0 & 78.47 & 1104.00 & 250.00 \\
\hline & Total: & & 6297.87 & 1274.92 & 6254.23 & 1387.10 \\
\hline
\end{tabular}


Table A34. Results of AC PF calculations in Case 7-1. AC branch data.

\begin{tabular}{|c|c|c|c|c|c|c|c|c|}
\hline \multirow{2}{*}{ Branch } & \multirow[b]{2}{*}{ From } & \multirow[b]{2}{*}{ To } & \multicolumn{2}{|c|}{ From Bus Injection } & \multicolumn{2}{|c|}{ To Bus Injection } & \multicolumn{2}{|c|}{ Power Losses } \\
\hline & & & $P$ & $Q$ & $P$ & $Q$ & $P$ & $Q$ \\
\hline 1 & 1 & 2 & -173.7 & -40.31 & 174.68 & -24.36 & 0.978 & 11.48 \\
\hline 2 & 1 & 39 & 76.10 & -3.89 & -76.03 & -74.75 & 0.066 & 1.65 \\
\hline 3 & 2 & 3 & 319.91 & 88.59 & -318.58 & -100.88 & 1.335 & 15.51 \\
\hline 4 & 2 & 25 & -244.59 & 82.97 & 248.93 & -93.84 & 4.337 & 5.33 \\
\hline 5 & 2 & 30 & -250.00 & -147.20 & 250.00 & 161.76 & 0.000 & 14.56 \\
\hline 6 & 3 & 4 & 37.34 & 113.06 & -37.13 & -132.59 & 0.208 & 3.40 \\
\hline 7 & 3 & 18 & -40.76 & -14.59 & 40.78 & -7.94 & 0.017 & 0.21 \\
\hline 8 & 4 & 5 & -197.45 & -4.09 & 197.76 & -4.52 & 0.309 & 4.95 \\
\hline 9 & 4 & 14 & -265.42 & -47.32 & 265.99 & 42.48 & 0.571 & 9.22 \\
\hline 10 & 5 & 6 & -536.94 & -43.11 & 537.51 & 46.16 & 0.573 & 7.45 \\
\hline 11 & 5 & 8 & 339.18 & 47.64 & -338.24 & -49.39 & 0.933 & 13.07 \\
\hline 12 & 6 & 7 & 453.82 & 81.55 & -452.56 & -73.59 & 1.261 & 19.33 \\
\hline 13 & 6 & 11 & -322.65 & -38.85 & 323.38 & 33.14 & 0.724 & 8.48 \\
\hline 14 & 6 & 31 & -668.67 & -88.85 & 668.67 & 216.97 & 0.000 & 128.12 \\
\hline 15 & 7 & 8 & 218.76 & -10.41 & -218.56 & 4.84 & 0.192 & 2.21 \\
\hline 16 & 8 & 9 & 34.81 & -132.06 & -34.48 & 97.72 & 0.324 & 5.11 \\
\hline 17 & 9 & 39 & 27.98 & -31.12 & -27.97 & -96.78 & 0.018 & 0.44 \\
\hline 18 & 10 & 11 & 327.90 & 73.37 & -327.46 & -76.18 & 0.438 & 4.71 \\
\hline 19 & 10 & 13 & 322.10 & 37.49 & -321.69 & -40.65 & 0.407 & 4.38 \\
\hline 20 & 10 & 32 & -650.00 & -110.87 & 650.00 & 206.96 & 0.000 & 96.1 \\
\hline 21 & 12 & 11 & -4.06 & -42.25 & 4.09 & 43.04 & 0.029 & 0.79 \\
\hline 22 & 12 & 13 & -4.47 & -45.75 & 4.51 & 46.68 & 0.034 & 0.93 \\
\hline 23 & 13 & 14 & 317.18 & -6.03 & -316.30 & -1.80 & 0.879 & 9.87 \\
\hline 24 & 14 & 15 & 50.31 & -40.68 & -50.26 & 3.66 & 0.053 & 0.64 \\
\hline 25 & 15 & 16 & -269.74 & -156.66 & 270.56 & 147.33 & 0.825 & 8.61 \\
\hline 26 & 16 & 17 & 224.02 & -42.54 & -223.68 & 32.50 & 0.338 & 4.29 \\
\hline 27 & 16 & 19 & -451.30 & -54.20 & 454.38 & 58.75 & 3.078 & 37.52 \\
\hline 28 & 16 & 21 & -329.60 & 14.44 & 330.42 & -27.74 & 0.821 & 13.86 \\
\hline 29 & 16 & 24 & -42.68 & -97.33 & 42.71 & 90.63 & 0.030 & 0.59 \\
\hline 30 & 17 & 18 & 199.04 & 11.05 & -198.78 & -22.06 & 0.261 & 3.06 \\
\hline 31 & 17 & 27 & 24.64 & -43.56 & -24.62 & 9.23 & 0.016 & 0.21 \\
\hline 32 & 19 & 20 & 174.73 & -9.170 & -174.51 & 13.48 & 0.218 & 4.30 \\
\hline 33 & 19 & 33 & -629.11 & -49.58 & 632.00 & 108.29 & 2.894 & 58.71 \\
\hline 34 & 20 & 34 & -505.49 & -116.48 & 508.00 & 166.69 & 2.511 & 50.21 \\
\hline 35 & 21 & 22 & -604.42 & -87.26 & 607.21 & 108.15 & 2.783 & 48.7 \\
\hline 36 & 22 & 23 & 42.790 & 41.88 & -42.77 & -61.75 & 0.025 & 0.40 \\
\hline 37 & 22 & 35 & -650.00 & -150.04 & 650.00 & 210.66 & 0.000 & 60.63 \\
\hline 38 & 23 & 24 & 353.84 & -0.50 & -351.31 & 1.57 & 2.529 & 40.24 \\
\hline 39 & 23 & 36 & -558.57 & -22.35 & 560.00 & 100.16 & 1.430 & 77.82 \\
\hline 40 & 25 & 26 & 65.41 & -18.81 & -65.29 & -39.04 & 0.126 & 1.27 \\
\hline 41 & 25 & 37 & -538.34 & 65.45 & 540.00 & -1.37 & 1.657 & 64.08 \\
\hline 42 & 26 & 27 & 257.30 & 68.21 & -256.38 & -84.73 & 0.920 & 9.66 \\
\hline 43 & 26 & 28 & -140.82 & -21.21 & 141.61 & -56.36 & 0.788 & 8.69 \\
\hline 44 & 26 & 29 & -190.19 & -24.96 & 192.10 & -67.79 & 1.914 & 20.98 \\
\hline 45 & 28 & 29 & -347.61 & 28.76 & 349.16 & -39.44 & 1.556 & 16.78 \\
\hline \multirow[t]{2}{*}{46} & 29 & 38 & -824.77 & 80.33 & 830.0 & 21.73 & 5.234 & 102.06 \\
\hline & & & & & & Total: & 43.640 & 1000.61 \\
\hline
\end{tabular}


Table A35. Results of Mixed AC/DC PF calculations in Case 7-2. AC bus data.

\begin{tabular}{|c|c|c|c|c|c|c|}
\hline \multirow{2}{*}{ Bus } & \multicolumn{2}{|c|}{ Voltage } & \multicolumn{2}{|c|}{ Generation } & \multicolumn{2}{|c|}{ Load } \\
\hline & $|V|$ (p.u.) & $\theta\left({ }^{\circ}\right)$ & $P$ & $Q$ & $P$ & $Q$ \\
\hline 1 & 1.047 & -12.439 & - & - & 97.60 & 44.20 \\
\hline 2 & 1.051 & -8.959 & - & - & - & - \\
\hline 3 & 1.034 & -11.548 & - & - & 322.00 & 2.400 \\
\hline 4 & 0.998 & -12.879 & - & - & 500.00 & 184.00 \\
\hline 5 & 1.001 & -11.443 & - & - & - & - \\
\hline 6 & 1.004 & -10.675 & - & - & - & - \\
\hline 7 & 0.994 & -12.969 & - & - & 233.80 & 84.00 \\
\hline 8 & 0.994 & -13.517 & - & - & 522.00 & 176.60 \\
\hline 9 & 1.037 & -13.804 & - & - & 6.50 & -66.60 \\
\hline 10 & 1.012 & -8.658 & - & - & - & - \\
\hline 11 & 1.009 & -9.390 & - & - & - & - \\
\hline 12 & 1.000 & -10.075 & - & - & 8.530 & 88.00 \\
\hline 13 & 1.008 & -9.464 & - & - & - & - \\
\hline 14 & 1.000 & -11.243 & - & - & - & - \\
\hline 15 & 1.016 & -10.879 & - & - & 320.00 & 153.00 \\
\hline 16 & 1.035 & -9.343 & - & - & 329.00 & 32.30 \\
\hline 17 & 1.040 & -10.213 & - & - & - & - \\
\hline 18 & 1.038 & -11.041 & - & - & 158.00 & 30.00 \\
\hline 19 & 1.051 & -4.727 & - & - & - & - \\
\hline 20 & 0.991 & -6.135 & - & - & 680.00 & 103.00 \\
\hline 21 & 1.034 & -6.945 & - & - & 274.00 & 115.00 \\
\hline 22 & 1.051 & -2.507 & - & - & - & - \\
\hline 23 & 1.046 & -2.705 & - & - & 247.50 & 84.60 \\
\hline 24 & 1.040 & -9.223 & - & - & 308.60 & -92.20 \\
\hline 25 & 1.060 & -7.519 & - & - & 224.00 & 47.20 \\
\hline 26 & 1.056 & -8.561 & - & - & 139.00 & 17.00 \\
\hline 27 & 1.043 & -10.465 & - & - & 281.00 & 75.50 \\
\hline 28 & 1.052 & -5.060 & - & - & 206.00 & 27.60 \\
\hline 29 & 1.051 & -2.306 & - & - & 283.50 & 26.90 \\
\hline 30 & 1.050 & -6.550 & 250.00 & 147.24 & - & - \\
\hline 31 & 0.982 & 0.000 & 691.88 & 240.39 & 9.20 & 4.600 \\
\hline 32 & 0.984 & -0.633 & 650.00 & 232.33 & - & - \\
\hline 33 & 0.997 & 0.488 & 632.00 & 103.03 & - & - \\
\hline 34 & 1.012 & -0.946 & 508.00 & 164.25 & - & - \\
\hline 35 & 1.049 & 2.449 & 650.00 & 204.75 & - & - \\
\hline 36 & 1.064 & 5.139 & 560.00 & 96.81 & - & - \\
\hline 37 & 1.028 & -0.744 & 540.00 & -10.62 & - & - \\
\hline 38 & 1.026 & 4.752 & 830.00 & 14.76 & - & - \\
\hline \multirow[t]{2}{*}{39} & 1.030 & -13.788 & 1000.0 & 54.67 & 1104.00 & 250.00 \\
\hline & Total: & & 6311.88 & 1247.61 & 6254.23 & 1387.10 \\
\hline
\end{tabular}


Table A36. Results of Mixed AC/DC PF calculations in Case 7-2. AC branch data.

\begin{tabular}{|c|c|c|c|c|c|c|c|c|}
\hline \multirow{2}{*}{ Branch } & \multirow[b]{2}{*}{ From } & \multirow[b]{2}{*}{ To } & \multicolumn{2}{|c|}{ From Bus Injection } & \multicolumn{2}{|c|}{ To Bus Injection } & \multicolumn{2}{|c|}{ Power Losses } \\
\hline & & & $P$ & $Q$ & $P$ & $Q$ & $P$ & $Q$ \\
\hline 1 & 1 & 2 & -161.82 & -30.43 & 162.66 & -36.59 & 0.838 & 9.85 \\
\hline 2 & 1 & 39 & 104.22 & 26.23 & -104.08 & -103.6 & 0.140 & 3.51 \\
\hline 3 & 2 & 3 & 333.45 & 80.70 & -332.03 & -92.24 & 1.414 & 16.43 \\
\hline 4 & 2 & 25 & -246.11 & 89.31 & 250.55 & -100.12 & 4.439 & 5.45 \\
\hline 5 & 2 & 30 & -250.00 & -133.42 & 250.00 & 147.24 & 0.000 & 13.82 \\
\hline 6 & 3 & 4 & 123.06 & 160.38 & -122.51 & -174.32 & 0.544 & 8.92 \\
\hline 7 & 3 & 18 & -73.03 & -30.54 & 73.08 & 8.30 & 0.059 & 0.71 \\
\hline 8 & 4 & 5 & -196.54 & -21.20 & 196.86 & 12.78 & 0.312 & 5.00 \\
\hline 9 & 4 & 14 & -220.94 & -8.49 & 221.34 & 1.030 & 0.392 & 6.33 \\
\hline 10 & 5 & 6 & -521.95 & -55.55 & 522.50 & 58.32 & 0.549 & 7.14 \\
\hline 11 & 5 & 8 & 325.09 & 42.76 & -324.23 & -45.37 & 0.863 & 12.09 \\
\hline 12 & 6 & 7 & 439.68 & 79.60 & -438.49 & -72.56 & 1.194 & 18.31 \\
\hline 13 & 6 & 11 & -279.50 & -37.36 & 280.05 & 29.73 & 0.549 & 6.43 \\
\hline 14 & 6 & 31 & -682.68 & -100.56 & 682.68 & 235.79 & 0.000 & 135.24 \\
\hline 15 & 7 & 8 & 204.69 & -11.44 & -204.52 & 5.68 & 0.170 & 1.95 \\
\hline 16 & 8 & 9 & 6.75 & -136.92 & -6.420 & 102.83 & 0.326 & 5.15 \\
\hline 17 & 9 & 39 & -0.08 & -36.23 & 0.08 & -91.73 & 0.007 & 0.19 \\
\hline 18 & 10 & 11 & 309.18 & 59.26 & -308.79 & -62.53 & 0.389 & 4.18 \\
\hline 19 & 10 & 13 & 340.82 & 74.67 & -340.34 & -76.98 & 0.477 & 5.13 \\
\hline 20 & 10 & 32 & -650.00 & -133.93 & 650.00 & 232.33 & 0.000 & 98.4 \\
\hline 21 & 12 & 11 & -28.72 & -31.98 & 28.75 & 32.80 & 0.030 & 0.81 \\
\hline 22 & 12 & 13 & -25.68 & -30.39 & 25.71 & 31.08 & 0.026 & 0.70 \\
\hline 23 & 13 & 14 & 314.63 & 45.89 & -313.73 & -53.12 & 0.904 & 10.14 \\
\hline 24 & 14 & 15 & -35.63 & -89.16 & 35.75 & 53.33 & 0.113 & 1.36 \\
\hline 25 & 15 & 16 & -315.75 & -176.33 & 316.86 & 169.98 & 1.114 & 11.63 \\
\hline 26 & 16 & 17 & 177.78 & -80.31 & -177.54 & 68.94 & 0.242 & 3.07 \\
\hline 27 & 16 & 19 & -451.33 & -47.15 & 454.39 & 51.36 & 3.059 & 37.28 \\
\hline 28 & 16 & 21 & -329.62 & 19.33 & 330.44 & -32.75 & 0.820 & 13.84 \\
\hline 29 & 16 & 24 & -42.68 & -94.16 & 42.71 & 87.39 & 0.028 & 0.55 \\
\hline 30 & 17 & 18 & 191.32 & 6.86 & -191.08 & -18.30 & 0.238 & 2.79 \\
\hline 31 & 17 & 27 & 26.22 & -35.79 & -26.20 & 1.090 & 0.012 & 0.16 \\
\hline 32 & 19 & 20 & 174.72 & -6.89 & -174.5 & 11.18 & 0.218 & 4.29 \\
\hline 33 & 19 & 33 & -629.11 & -44.47 & 632.00 & 103.03 & 2.886 & 58.55 \\
\hline 34 & 20 & 34 & -505.50 & -114.18 & 508.00 & 164.25 & 2.503 & 50.07 \\
\hline 35 & 21 & 22 & -604.44 & -82.25 & 607.21 & 102.85 & 2.770 & 48.47 \\
\hline 36 & 22 & 23 & 42.79 & 41.60 & -42.76 & -61.50 & 0.025 & 0.39 \\
\hline 37 & 22 & 35 & -650.00 & -144.44 & 650.00 & 204.75 & 0.000 & 60.31 \\
\hline 38 & 23 & 24 & 353.84 & -3.95 & -351.31 & 4.81 & 2.522 & 40.13 \\
\hline 39 & 23 & 36 & -558.57 & -19.16 & 560.00 & 96.81 & 1.428 & 77.66 \\
\hline 40 & 25 & 26 & 63.79 & -21.80 & -63.68 & -36.42 & 0.118 & 1.19 \\
\hline 41 & 25 & 37 & -538.34 & 74.72 & 540.00 & -10.62 & 1.658 & 64.10 \\
\hline 42 & 26 & 27 & 255.68 & 59.54 & -254.8 & -76.59 & 0.888 & 9.32 \\
\hline 43 & 26 & 28 & -140.79 & -18.17 & 141.58 & -59.77 & 0.790 & 8.70 \\
\hline 44 & 26 & 29 & -190.22 & -21.95 & 192.14 & -71.24 & 1.915 & 21.00 \\
\hline 45 & 28 & 29 & -347.58 & 32.17 & 349.13 & -42.93 & 1.555 & 16.77 \\
\hline \multirow[t]{2}{*}{46} & 29 & 38 & -824.77 & 87.27 & 830.00 & 14.76 & 5.232 & 102.02 \\
\hline & & & & & & Total: & 43.756 & 1009.53 \\
\hline
\end{tabular}


Table A37. Results of the mixed AC/DC PF calculations in Case 7-2. DC bus data.

\begin{tabular}{cccc}
\hline \multirow{2}{*}{ Bus DC } & \multicolumn{2}{c}{ Bus Injection } & Total Loss \\
\cline { 2 - 4 } & $\boldsymbol{P}$ & $\boldsymbol{Q}$ & $\boldsymbol{P}$ \\
\hline 1 & -40.00 & -20.00 & 1.24 \\
2 & -45.87 & 25.63 & 1.25 \\
3 & 40.00 & 40.00 & 1.24 \\
4 & 40.00 & 40.00 & 1.24 \\
5 & -128.03 & -141.24 & 2.44 \\
6 & 40.00 & 30.00 & 1.22 \\
7 & 40.00 & 20.00 & 1.20 \\
8 & 40.00 & 40.00 & 1.24 \\
& Total: & & 11.07 \\
\hline
\end{tabular}

Table A38. Results of the mixed AC/DC PF calculations in Case 7-2. DC branch data.

\begin{tabular}{|c|c|c|c|c|c|}
\hline \multirow{2}{*}{ Branch } & \multicolumn{3}{|c|}{ From Bus } & \multirow{2}{*}{$\begin{array}{c}\text { To Bus } \\
P\end{array}$} & \multirow{2}{*}{$\begin{array}{c}\text { Power Losses } \\
P\end{array}$} \\
\hline & From & To & $P$ & & \\
\hline 1 & 1 & 2 & -2.19 & 2.19 & 0.00 \\
\hline 2 & 1 & 4 & 40.95 & -40.51 & 0.44 \\
\hline 3 & 2 & 3 & 42.43 & -41.96 & 0.47 \\
\hline 4 & 3 & 4 & 0.72 & -0.72 & 0.00 \\
\hline 5 & 5 & 6 & 41.86 & -41.22 & 0.64 \\
\hline 6 & 5 & 7 & 41.85 & -41.21 & 0.64 \\
\hline 7 & 5 & 8 & 41.87 & -41.23 & 0.64 \\
\hline 8 & 6 & 7 & -0.01 & 0.01 & 0.00 \\
\hline \multirow[t]{2}{*}{9} & 6 & 8 & 0.01 & -0.01 & 0.00 \\
\hline & & & & Total: & 2.83 \\
\hline
\end{tabular}

Table A39. Droop control settings for each converter station.

\begin{tabular}{cccc}
\hline Converter Station & Droop Parameter & $\boldsymbol{P}_{\boldsymbol{D} C}^{*}(\mathbf{M W})$ & $\boldsymbol{V}_{\boldsymbol{D C}}^{*}$ (p.u.) \\
\hline 1 & 0.0017 & -50.0000 & 1.0079 \\
2 & 0.0017 & -35.0000 & 1.0000 \\
3 & 0.0017 & 60.0000 & 1.0000 \\
4 & 0.0017 & 60.0000 & 1.0000 \\
5 & 0.0017 & -35.0000 & 1.0000 \\
6 & 0.0017 & 40.0000 & 1.0000 \\
7 & 0.0017 & 40.0000 & 1.0000 \\
8 & 0.0017 & 40.0000 & 1.0000 \\
\hline
\end{tabular}

\footnotetext{
${ }^{*} P_{D C}^{*}$ and $V_{D C}^{*}$ are the reference power and reference DC voltage, respectively.
} 
Table A40. Results of Mixed AC/DC PF calculations in Case 7-3. AC bus data.

\begin{tabular}{|c|c|c|c|c|c|c|}
\hline \multirow{2}{*}{ Bus } & \multicolumn{2}{|c|}{ Voltage } & \multicolumn{2}{|c|}{ Generation } & \multicolumn{2}{|c|}{ Load } \\
\hline & $|V|$ (p.u.) & $\theta\left({ }^{\circ}\right)$ & $P$ & $Q$ & $P$ & $Q$ \\
\hline 1 & 1.047 & -12.504 & - & - & 97.60 & 44.20 \\
\hline 2 & 1.051 & -9.194 & - & - & - & - \\
\hline 3 & 1.034 & -11.802 & - & - & 322.00 & 2.40 \\
\hline 4 & 0.997 & -12.917 & - & - & 500.00 & 184.00 \\
\hline 5 & 1.001 & -11.426 & - & - & - & - \\
\hline 6 & 1.004 & -10.647 & - & - & - & - \\
\hline 7 & 0.994 & -12.949 & - & - & 233.80 & 84.00 \\
\hline 8 & 0.994 & -13.501 & - & - & 522.00 & 176.60 \\
\hline 9 & 1.037 & -13.823 & - & - & 6.50 & -66.60 \\
\hline 10 & 1.012 & -8.558 & - & - & - & - \\
\hline 11 & 1.009 & -9.310 & - & - & - & - \\
\hline 12 & 1.000 & -9.926 & - & - & 8.530 & 88.00 \\
\hline 13 & 1.008 & -9.343 & - & - & - & - \\
\hline 14 & 1.000 & -11.08 & - & - & - & - \\
\hline 15 & 1.016 & -11.186 & - & - & 320.00 & 153.00 \\
\hline 16 & 1.034 & -9.727 & - & - & 329.00 & 32.30 \\
\hline 17 & 1.04 & -10.668 & - & - & - & - \\
\hline 18 & 1.037 & -11.483 & - & - & 158.00 & 30.00 \\
\hline 19 & 1.051 & -5.110 & - & - & - & - \\
\hline 20 & 0.991 & -6.519 & - & - & 680.00 & 103.00 \\
\hline 21 & 1.034 & -7.328 & - & - & 274.00 & 115.00 \\
\hline 22 & 1.051 & -2.889 & - & - & - & - \\
\hline 23 & 1.046 & -3.087 & - & - & 247.50 & 84.60 \\
\hline 24 & 1.040 & -9.607 & - & - & 308.60 & -92.20 \\
\hline 25 & 1.060 & -7.783 & - & - & 224.00 & 47.20 \\
\hline 26 & 1.055 & -8.920 & - & - & 139.00 & 17.00 \\
\hline 27 & 1.042 & -10.869 & - & - & 281.00 & 75.50 \\
\hline 28 & 1.052 & -5.418 & - & - & 206.00 & 27.60 \\
\hline 29 & 1.051 & -2.664 & - & - & 283.50 & 26.90 \\
\hline 30 & 1.050 & -6.785 & 250.00 & 146.82 & - & - \\
\hline 31 & 0.982 & 0.000 & 689.99 & 240.44 & 9.200 & 4.60 \\
\hline 32 & 0.984 & -0.532 & 650.00 & 232.48 & - & - \\
\hline 33 & 0.997 & 0.105 & 632.00 & 103.62 & - & - \\
\hline 34 & 1.012 & -1.329 & 508.00 & 164.52 & - & - \\
\hline 35 & 1.049 & 2.067 & 650.00 & 205.41 & - & - \\
\hline 36 & 1.064 & 4.758 & 560.00 & 97.19 & - & - \\
\hline 37 & 1.028 & -1.007 & 540.00 & -9.75 & - & - \\
\hline 38 & 1.027 & 4.395 & 830.00 & 15.39 & - & - \\
\hline \multirow[t]{2}{*}{39} & 1.030 & -13.830 & 1000.00 & 54.04 & 1104.00 & 250.00 \\
\hline & Total: & & 6309.99 & 1250.16 & 6254.23 & 1387.10 \\
\hline
\end{tabular}


Table A41. Results of Mixed AC/DC PF calculations in Case 7-3. AC branch data.

\begin{tabular}{|c|c|c|c|c|c|c|c|c|}
\hline \multirow{2}{*}{ Branch } & \multirow[b]{2}{*}{ From } & \multirow[b]{2}{*}{ To } & \multicolumn{2}{|c|}{ From Bus Injection } & \multicolumn{2}{|c|}{ To Bus Injection } & \multicolumn{2}{|c|}{ Power Losses } \\
\hline & & & $P$ & $Q$ & $P$ & $Q$ & $P$ & $Q$ \\
\hline 1 & 1 & 2 & -154.00 & -31.28 & 154.76 & -36.7 & 0.759 & 8.91 \\
\hline 2 & 1 & 39 & 102.52 & 27.08 & -102.38 & -104.51 & 0.138 & 3.46 \\
\hline 3 & 2 & 3 & 335.86 & 81.69 & -334.43 & -92.98 & 1.435 & 16.67 \\
\hline 4 & 2 & 25 & -240.62 & 88.03 & 244.87 & -99.07 & 4.253 & 5.23 \\
\hline 5 & 2 & 30 & -250.00 & -133.01 & 250.00 & 146.82 & 0.000 & 13.8 \\
\hline 6 & 3 & 4 & 104.84 & 161.43 & -104.34 & -176.12 & 0.498 & 8.17 \\
\hline 7 & 3 & 18 & -46.55 & -30.85 & 46.57 & 8.23 & 0.026 & 0.32 \\
\hline 8 & 4 & 5 & -203.98 & -20.74 & 204.32 & 12.72 & 0.336 & 5.38 \\
\hline 9 & 4 & 14 & -247.94 & -7.14 & 248.43 & 1.33 & 0.494 & 7.97 \\
\hline 10 & 5 & 6 & -529.57 & -55.39 & 530.13 & 58.38 & 0.565 & 7.35 \\
\hline 11 & 5 & 8 & 325.25 & 42.68 & -324.39 & -45.26 & 0.864 & 12.1 \\
\hline 12 & 6 & 7 & 441.24 & 79.61 & -440.04 & -72.45 & 1.203 & 18.44 \\
\hline 13 & 6 & 11 & -290.58 & -36.72 & 291.17 & 29.61 & 0.593 & 6.94 \\
\hline 14 & 6 & 31 & -680.79 & -101.27 & 680.79 & 235.84 & 0.000 & 134.58 \\
\hline 15 & 7 & 8 & 206.24 & -11.55 & -206.07 & 5.83 & 0.172 & 1.98 \\
\hline 16 & 8 & 9 & 8.45 & -137.17 & -8.12 & 103.12 & 0.328 & 5.18 \\
\hline 17 & 9 & 39 & 1.62 & -36.52 & -1.62 & -91.44 & 0.007 & 0.18 \\
\hline 18 & 10 & 11 & 317.57 & 59.28 & -317.16 & -62.33 & 0.409 & 4.4 \\
\hline 19 & 10 & 13 & 332.43 & 74.78 & -331.97 & -77.32 & 0.455 & 4.9 \\
\hline 20 & 10 & 32 & -650.00 & -134.06 & 650.00 & 232.48 & 0.000 & 98.41 \\
\hline 21 & 12 & 11 & -25.96 & -31.97 & 25.99 & 32.72 & 0.027 & 0.75 \\
\hline 22 & 12 & 13 & -24.57 & -30.42 & 24.59 & 31.09 & 0.025 & 0.67 \\
\hline 23 & 13 & 14 & 307.38 & 46.23 & -306.52 & -53.90 & 0.864 & 9.7 \\
\hline 24 & 14 & 15 & 2.66 & -91.24 & -2.56 & 55.21 & 0.096 & 1.16 \\
\hline 25 & 15 & 16 & -300.93 & -178.21 & 301.97 & 171.1 & 1.040 & 10.86 \\
\hline 26 & 16 & 17 & 192.66 & -79.73 & -192.39 & 68.82 & 0.277 & 3.52 \\
\hline 27 & 16 & 19 & -451.33 & -47.94 & 454.39 & 52.19 & 3.061 & 37.3 \\
\hline 28 & 16 & 21 & -329.62 & 18.78 & 330.44 & -32.19 & 0.820 & 13.84 \\
\hline 29 & 16 & 24 & -42.68 & -94.52 & 42.71 & 87.76 & 0.028 & 0.56 \\
\hline 30 & 17 & 18 & 188.28 & 6.71 & -188.04 & -18.23 & 0.231 & 2.7 \\
\hline 31 & 17 & 27 & 20.60 & -35.53 & -20.59 & 0.80 & 0.009 & 0.12 \\
\hline 32 & 19 & 20 & 174.72 & -7.14 & -174.50 & 11.44 & 0.218 & 4.29 \\
\hline 33 & 19 & 33 & -629.11 & -45.05 & 632.00 & 103.62 & 2.887 & 58.57 \\
\hline 34 & 20 & 34 & -505.50 & -114.44 & 508.00 & 164.52 & 2.504 & 50.08 \\
\hline 35 & 21 & 22 & -604.44 & -82.81 & 607.21 & 103.44 & 2.771 & 48.5 \\
\hline 36 & 22 & 23 & 42.79 & 41.63 & -42.76 & -61.53 & 0.025 & 0.39 \\
\hline 37 & 22 & 35 & -650.00 & -145.07 & 650.00 & 205.41 & 0.000 & 60.34 \\
\hline 38 & 23 & 24 & 353.84 & -3.56 & -351.31 & 4.44 & 2.523 & 40.14 \\
\hline 39 & 23 & 36 & -558.57 & -19.51 & 560.00 & 97.19 & 1.428 & 77.67 \\
\hline 40 & 25 & 26 & 69.47 & -21.98 & -69.33 & -35.99 & 0.139 & 1.41 \\
\hline 41 & 25 & 37 & -538.34 & 73.85 & 540.00 & -9.75 & 1.658 & 64.1 \\
\hline 42 & 26 & 27 & 261.34 & 59.66 & -260.41 & -76.30 & 0.926 & 9.72 \\
\hline 43 & 26 & 28 & -140.79 & -18.45 & 141.58 & -59.46 & 0.789 & 8.70 \\
\hline 44 & 26 & 29 & -190.22 & -22.23 & 192.13 & -70.92 & 1.915 & 21.0 \\
\hline 45 & 28 & 29 & -347.58 & 31.86 & 349.13 & -42.61 & 1.555 & 16.77 \\
\hline \multirow[t]{2}{*}{46} & 29 & 38 & -824.77 & 86.63 & 830.00 & 15.39 & 5.232 & 102.03 \\
\hline & & & & & & Total: & 43.583 & 1009.26 \\
\hline
\end{tabular}


Table A42. Results of the mixed AC/DC PF calculations in Case 7-3. DC bus data.

\begin{tabular}{cccc}
\hline \multirow{2}{*}{ Bus DC } & \multicolumn{2}{c}{ Bus Injection } & Total Loss \\
\cline { 2 - 4 } & $\boldsymbol{P}$ & $\boldsymbol{Q}$ & $\boldsymbol{P}$ \\
\hline 1 & -56.26 & -20.00 & 1.30 \\
2 & -42.00 & 25.61 & 1.23 \\
3 & 45.86 & 40.00 & 1.26 \\
4 & 46.12 & 40.00 & 1.26 \\
5 & -55.43 & -143.80 & 2.04 \\
6 & 16.51 & 30.00 & 1.16 \\
7 & 16.53 & 20.00 & 1.14 \\
8 & 16.48 & 40.00 & 1.19 \\
& \multicolumn{3}{c}{ Total: } \\
\end{tabular}

Table A43. Results of the mixed AC/DC PF calculations in Case 7-3. DC branch data.

\begin{tabular}{|c|c|c|c|c|c|}
\hline \multirow{2}{*}{ Branch } & \multicolumn{3}{|c|}{ From Bus } & \multirow{2}{*}{$\begin{array}{c}\text { To Bus } \\
P\end{array}$} & \multirow{2}{*}{$\begin{array}{c}\text { Power Losses } \\
P\end{array}$} \\
\hline & From & To & $P$ & & \\
\hline 1 & 1 & 2 & 5.27 & -5.26 & 0.01 \\
\hline 2 & 1 & 4 & 49.69 & -49.03 & 0.65 \\
\hline 3 & 2 & 3 & 46.03 & -45.47 & 0.56 \\
\hline 4 & 3 & 4 & -1.65 & 1.65 & 0.00 \\
\hline 5 & 5 & 6 & 17.79 & -17.67 & 0.12 \\
\hline 6 & 5 & 7 & 17.79 & -17.67 & 0.12 \\
\hline 7 & 5 & 8 & 17.79 & -17.67 & 0.12 \\
\hline 8 & 6 & 7 & 0.00 & 0.00 & 0.00 \\
\hline \multirow[t]{2}{*}{9} & 6 & 8 & 0.00 & 0.00 & 0.00 \\
\hline & & & & Total: & 1.58 \\
\hline
\end{tabular}


Table A44. Results of Mixed AC/DC PF calculations in Case 7-4. AC bus data.

\begin{tabular}{|c|c|c|c|c|c|c|}
\hline \multirow{2}{*}{ Bus } & \multicolumn{2}{|c|}{ Voltage } & \multicolumn{2}{|c|}{ Generation } & \multicolumn{2}{|c|}{ Load } \\
\hline & $|V|$ (p.u.) & $\theta\left({ }^{\circ}\right)$ & $P$ & $Q$ & $P$ & $Q$ \\
\hline 1 & 1.043 & -15.383 & - & - & 97.60 & 44.20 \\
\hline 2 & 1.058 & -11.984 & - & - & - & - \\
\hline 3 & 1.055 & -14.490 & - & - & 322.00 & 2.40 \\
\hline 4 & 0.932 & -16.184 & - & - & 500.00 & 184.00 \\
\hline 5 & 0.963 & -12.185 & - & - & - & - \\
\hline 6 & 0.971 & -10.906 & - & - & - & - \\
\hline 7 & 0.961 & -13.647 & - & - & 233.80 & 84.00 \\
\hline 8 & 0.960 & -14.378 & - & - & 522.00 & 176.60 \\
\hline 9 & 1.023 & -15.624 & - & - & 6.50 & -66.6 \\
\hline 10 & 0.995 & -6.307 & - & - & - & - \\
\hline 11 & 0.985 & -7.818 & - & - & - & - \\
\hline 12 & 0.975 & -7.165 & - & - & 8.53 & 88.00 \\
\hline 13 & 0.993 & -6.380 & - & - & - & - \\
\hline 14 & 0.994 & -6.385 & - & - & - & - \\
\hline 15 & 1.016 & -14.260 & - & - & 320.00 & 153.00 \\
\hline 16 & 1.033 & -12.688 & - & - & 329.00 & 32.30 \\
\hline 17 & 1.035 & -13.530 & - & - & - & - \\
\hline 18 & 1.032 & -14.217 & - & - & 158.00 & 30.00 \\
\hline 19 & 1.050 & -8.066 & - & - & - & - \\
\hline 20 & 0.991 & -9.477 & - & - & 680.00 & 103.00 \\
\hline 21 & 1.032 & -10.284 & - & - & 274.00 & 115.00 \\
\hline 22 & 1.050 & -5.839 & - & - & - & - \\
\hline 23 & 1.045 & -6.037 & - & - & 247.50 & 84.60 \\
\hline 24 & 1.038 & -12.569 & - & - & 308.60 & -92.20 \\
\hline 25 & 1.064 & -10.503 & - & - & 224.00 & 47.20 \\
\hline 26 & 1.055 & -11.705 & - & - & 139.00 & 17.00 \\
\hline 27 & 1.040 & -13.691 & - & - & 281.00 & 75.50 \\
\hline 28 & 1.052 & -8.202 & - & - & 206.00 & 27.60 \\
\hline 29 & 1.051 & -5.447 & - & - & 283.50 & 26.90 \\
\hline 30 & 1.050 & -9.591 & 250.00 & 108.31 & - & - \\
\hline 31 & 0.982 & 0.000 & 683.41 & 362.78 & 9.20 & 4.60 \\
\hline 32 & 0.984 & 1.860 & 650.00 & 313.16 & - & - \\
\hline 33 & 0.997 & -2.849 & 632.00 & 107.87 & - & - \\
\hline 34 & 1.012 & -4.287 & 508.00 & 166.49 & - & - \\
\hline 35 & 1.049 & -0.880 & 650.00 & 210.19 & - & - \\
\hline 36 & 1.064 & 1.812 & 560.00 & 99.90 & - & - \\
\hline 37 & 1.028 & -3.746 & 540.00 & -26.63 & - & - \\
\hline 38 & 1.027 & 1.612 & 830.00 & 16.42 & - & - \\
\hline \multirow[t]{2}{*}{39} & 1.030 & -16.197 & 1000.00 & 126.31 & 1104.00 & 250.00 \\
\hline & Total: & & 6303.41 & 1484.80 & 6254.23 & 1387.10 \\
\hline
\end{tabular}


Table A45. Results of Mixed AC/DC PF calculations in Case 7-4. AC branch data.

\begin{tabular}{|c|c|c|c|c|c|c|c|c|}
\hline \multirow{2}{*}{ Branch } & \multirow[b]{2}{*}{ From } & \multirow[b]{2}{*}{ To } & \multicolumn{2}{|c|}{ From Bus Injection } & \multicolumn{2}{|c|}{ To Bus Injection } & \multicolumn{2}{|c|}{ Power Losses } \\
\hline & & & $P$ & $Q$ & $P$ & $Q$ & $P$ & $Q$ \\
\hline 1 & 1 & 2 & -160.79 & -56.77 & 161.63 & -10.46 & 0.843 & 9.90 \\
\hline 2 & 1 & 39 & 63.19 & 12.57 & -63.13 & -91.60 & 0.063 & 1.57 \\
\hline 3 & 2 & 3 & 323.21 & -12.20 & -322.00 & -2.40 & 1.213 & 14.09 \\
\hline 4 & 2 & 25 & -234.85 & 118.79 & 239.31 & -129.74 & 4.458 & 5.48 \\
\hline 5 & 2 & 30 & -250.00 & -96.12 & 250.00 & 108.31 & 0.000 & 12.19 \\
\hline 6 & 3 & 4 & 0.00 & 0.00 & 0.00 & 0.00 & 0.000 & 0.00 \\
\hline 7 & 3 & 18 & 0.00 & 0.00 & 0.00 & 0.00 & 0.000 & 0.00 \\
\hline 8 & 4 & 5 & -500.00 & -184.00 & 502.60 & 213.48 & 2.595 & 41.53 \\
\hline 9 & 4 & 14 & 0.00 & 0.00 & 0.00 & 0.00 & 0.000 & 0.00 \\
\hline 10 & 5 & 6 & -819.04 & -214.86 & 820.59 & 230.88 & 1.544 & 20.08 \\
\hline 11 & 5 & 8 & 316.45 & 1.38 & -315.58 & -2.93 & 0.864 & 12.10 \\
\hline 12 & 6 & 7 & 490.24 & 81.71 & -488.66 & -68.05 & 1.579 & 24.20 \\
\hline 13 & 6 & 11 & -636.61 & -105.52 & 639.69 & 128.36 & 3.084 & 36.12 \\
\hline 14 & 6 & 31 & -674.21 & -207.08 & 674.21 & 358.18 & 0.000 & 151.11 \\
\hline 15 & 7 & 8 & 254.86 & -15.95 & -254.58 & 12.01 & 0.282 & 3.25 \\
\hline 16 & 8 & 9 & 48.16 & -185.68 & -47.40 & 160.29 & 0.763 & 12.05 \\
\hline 17 & 9 & 39 & 40.90 & -93.69 & -40.87 & -32.10 & 0.025 & 0.63 \\
\hline 18 & 10 & 11 & 617.69 & 175.82 & -616.01 & -165.00 & 1.672 & 17.97 \\
\hline 19 & 10 & 13 & 32.31 & 29.83 & -32.31 & -36.94 & 0.009 & 0.09 \\
\hline 20 & 10 & 32 & -650.00 & -205.65 & 650.00 & 313.16 & 0.000 & 107.51 \\
\hline 21 & 12 & 11 & 23.71 & -35.79 & -23.68 & 36.64 & 0.031 & 0.85 \\
\hline 22 & 12 & 13 & -32.24 & -52.21 & 32.31 & 53.95 & 0.064 & 1.74 \\
\hline 23 & 13 & 14 & 0.00 & -17.01 & 0.00 & 0.00 & 0.001 & 0.01 \\
\hline 24 & 14 & 15 & 0.00 & 0.00 & 0.00 & 0.00 & 0.000 & 0.00 \\
\hline 25 & 15 & 16 & -320.00 & -153.00 & 321.07 & 146.27 & 1.074 & 11.21 \\
\hline 26 & 16 & 17 & 173.51 & -42.68 & -173.31 & 30.96 & 0.206 & 2.62 \\
\hline 27 & 16 & 19 & -451.30 & -53.64 & 454.38 & 58.17 & 3.077 & 37.50 \\
\hline 28 & 16 & 21 & -329.60 & 14.83 & 330.42 & -28.14 & 0.821 & 13.85 \\
\hline 29 & 16 & 24 & -42.68 & -97.08 & 42.71 & 90.37 & 0.030 & 0.58 \\
\hline 30 & 17 & 18 & 158.17 & 17.88 & -158.00 & -30.00 & 0.168 & 1.96 \\
\hline 31 & 17 & 27 & 15.14 & -48.84 & -15.12 & 14.44 & 0.015 & 0.20 \\
\hline 32 & 19 & 20 & 174.73 & -8.99 & -174.51 & 13.30 & 0.218 & 4.30 \\
\hline 33 & 19 & 33 & -629.11 & -49.17 & 632.00 & 107.87 & 2.894 & 58.70 \\
\hline 34 & 20 & 34 & -505.49 & -116.30 & 508.00 & 166.49 & 2.510 & 50.20 \\
\hline 35 & 21 & 22 & -604.42 & -86.86 & 607.21 & 107.73 & 2.782 & 48.69 \\
\hline 36 & 22 & 23 & 42.79 & 41.86 & -42.77 & -61.73 & 0.025 & 0.40 \\
\hline 37 & 22 & 35 & -650.00 & -149.59 & 650.00 & 210.19 & 0.000 & 60.60 \\
\hline 38 & 23 & 24 & 353.84 & -0.77 & -351.31 & 1.83 & 2.529 & 40.23 \\
\hline 39 & 23 & 36 & -558.57 & -22.10 & 560.00 & 99.90 & 1.430 & 77.80 \\
\hline 40 & 25 & 26 & 75.03 & -8.33 & -74.86 & -49.50 & 0.173 & 1.74 \\
\hline 41 & 25 & 37 & -538.34 & 90.86 & 540.00 & -26.63 & 1.661 & 64.23 \\
\hline 42 & 26 & 27 & 266.87 & 74.07 & -265.88 & -89.94 & 0.992 & 10.42 \\
\hline 43 & 26 & 28 & -140.79 & -18.90 & 141.58 & -58.95 & 0.789 & 8.70 \\
\hline 44 & 26 & 29 & -190.21 & -22.67 & 192.13 & -70.41 & 1.915 & 20.99 \\
\hline 45 & 28 & 29 & -347.58 & 31.35 & 349.14 & -42.10 & 1.555 & 16.78 \\
\hline \multirow[t]{2}{*}{46} & 29 & 38 & -824.77 & 85.61 & 830.00 & 16.42 & 5.232 & 102.03 \\
\hline & & & & & & Total: & 49.186 & 1106.20 \\
\hline
\end{tabular}


Table A46. Results of Mixed AC/DC PF calculations in Case 7-5. AC bus data.

\begin{tabular}{|c|c|c|c|c|c|c|}
\hline \multirow{2}{*}{ Bus } & \multicolumn{2}{|c|}{ Voltage } & \multicolumn{2}{|c|}{ Generation } & \multicolumn{2}{|c|}{ Load } \\
\hline & $|V|$ (p.u.) & $\theta\left({ }^{\circ}\right)$ & $P$ & $Q$ & $P$ & $Q$ \\
\hline 1 & 1.046 & -5.461 & - & - & 97.60 & 44.20 \\
\hline 2 & 1.060 & 1.265 & - & - & - & - \\
\hline 3 & 1.063 & -0.937 & - & - & 322.00 & 2.40 \\
\hline 4 & 0.934 & -16.570 & - & - & 500.00 & 184.00 \\
\hline 5 & 0.969 & -12.289 & - & - & - & - \\
\hline 6 & 0.977 & -11.124 & - & - & - & - \\
\hline 7 & 0.967 & -13.306 & - & - & 233.80 & 84.00 \\
\hline 8 & 0.966 & -13.764 & - & - & 522.00 & 176.60 \\
\hline 9 & 1.025 & -10.911 & - & - & 6.50 & -66.60 \\
\hline 10 & 1.003 & -7.787 & - & - & - & - \\
\hline 11 & 0.994 & -8.935 & - & - & - & - \\
\hline 12 & 1.000 & -9.246 & - & - & 8.53 & 88.00 \\
\hline 13 & 1.002 & -8.201 & - & - & - & - \\
\hline 14 & 1.000 & -8.935 & - & - & - & - \\
\hline 15 & 1.032 & 4.023 & - & - & 320.00 & 153.00 \\
\hline 16 & 1.045 & 5.368 & - & - & 329.00 & 32.30 \\
\hline 17 & 1.051 & 4.342 & - & - & - & - \\
\hline 18 & 1.050 & 3.841 & - & - & 158.00 & 30.00 \\
\hline 19 & 1.055 & 9.953 & - & - & - & - \\
\hline 20 & 0.993 & 8.557 & - & - & 680.00 & 103.00 \\
\hline 21 & 1.041 & 7.738 & - & - & 274.00 & 115.00 \\
\hline 22 & 1.055 & 12.140 & - & - & - & - \\
\hline 23 & 1.050 & 11.944 & - & - & 247.50 & 84.60 \\
\hline 24 & 1.049 & 5.488 & - & - & 308.60 & -92.20 \\
\hline 25 & 1.070 & 3.371 & - & - & 224.00 & 47.20 \\
\hline 26 & 1.064 & 4.157 & - & - & 139.00 & 17.00 \\
\hline 27 & 1.053 & 3.112 & - & - & 281.00 & 75.50 \\
\hline 28 & 1.057 & 7.633 & - & - & 206.00 & 27.60 \\
\hline 29 & 1.054 & 10.373 & - & - & 283.50 & 26.90 \\
\hline 30 & 1.050 & 3.655 & 250.00 & 99.19 & - & - \\
\hline 31 & 0.982 & 0.000 & 701.04 & 343.28 & 9.20 & 4.60 \\
\hline 32 & 0.984 & 0.317 & 650.00 & 277.42 & - & - \\
\hline 33 & 0.997 & 15.160 & 632.00 & 78.96 & - & - \\
\hline 34 & 1.012 & 13.742 & 508.00 & 153.10 & - & - \\
\hline 35 & 1.049 & 17.078 & 650.00 & 177.74 & - & - \\
\hline 36 & 1.064 & 19.762 & 560.00 & 81.49 & - & - \\
\hline 37 & 1.028 & 10.095 & 540.00 & -55.46 & - & - \\
\hline 38 & 1.026 & 17.419 & 830.00 & -5.42 & - & - \\
\hline \multirow[t]{2}{*}{39} & 1.030 & -8.849 & 1000.00 & 115.54 & 1104.00 & 250.00 \\
\hline & Total: & & 6321.04 & 1265.84 & 6254.23 & 1387.10 \\
\hline
\end{tabular}


Table A47. Results of Mixed AC/DC PF calculations in Case 7-5. AC branch data.

\begin{tabular}{|c|c|c|c|c|c|c|c|c|}
\hline \multirow{2}{*}{ Branch } & \multirow[b]{2}{*}{ From } & \multirow[b]{2}{*}{ To } & \multicolumn{2}{|c|}{ From Bus Injection } & \multicolumn{2}{|c|}{ To Bus Injection } & \multicolumn{2}{|c|}{ Power Losses } \\
\hline & & & $P$ & $Q$ & $P$ & $Q$ & $P$ & $Q$ \\
\hline 1 & 1 & 2 & -314.89 & -27.33 & 318.06 & -12.82 & 3.176 & 37.29 \\
\hline 2 & 1 & 39 & 257.29 & 23.13 & -256.65 & -87.88 & 0.643 & 16.07 \\
\hline 3 & 2 & 3 & 282.95 & -55.56 & -282.00 & 37.60 & 0.947 & 11.00 \\
\hline 4 & 2 & 25 & -351.01 & 155.69 & 360.37 & -160.75 & 9.357 & 11.50 \\
\hline 5 & 2 & 30 & -250.00 & -87.31 & 250.00 & 99.19 & 0.000 & 11.88 \\
\hline 6 & 3 & 4 & 0.00 & 0.00 & 0.00 & 0.00 & 0.000 & 0.00 \\
\hline 7 & 3 & 18 & 0.00 & 0.00 & 0.00 & 0.00 & 0.000 & 0.00 \\
\hline 8 & 4 & 5 & -540.00 & -204.00 & 543.03 & 240.39 & 3.034 & 48.54 \\
\hline 9 & 4 & 14 & 0.00 & 0.00 & 0.00 & 0.00 & 0.000 & 0.000 \\
\hline 10 & 5 & 6 & -758.72 & -243.95 & 760.07 & 257.42 & 1.352 & 17.58 \\
\hline 11 & 5 & 8 & 215.68 & 3.56 & -215.29 & -11.80 & 0.398 & 5.57 \\
\hline 12 & 6 & 7 & 396.78 & 85.56 & -395.74 & -80.25 & 1.042 & 15.98 \\
\hline 13 & 6 & 11 & -465.01 & -158.13 & 466.76 & 165.20 & 1.755 & 20.55 \\
\hline 14 & 6 & 31 & -691.84 & -184.85 & 691.84 & 338.68 & 0.000 & 153.82 \\
\hline 15 & 7 & 8 & 161.94 & -3.75 & -161.82 & -2.25 & 0.112 & 1.29 \\
\hline 16 & 8 & 9 & -144.89 & -162.55 & 145.92 & 141.15 & 1.034 & 16.33 \\
\hline 17 & 9 & 39 & -152.42 & -74.55 & 152.65 & -46.59 & 0.222 & 5.56 \\
\hline 18 & 10 & 11 & 480.08 & 167.66 & -479.05 & -163.81 & 1.034 & 11.11 \\
\hline 19 & 10 & 13 & 169.92 & 6.61 & -169.8 & -12.69 & 0.115 & 1.24 \\
\hline 20 & 10 & 32 & -650.00 & -174.27 & 650.00 & 277.42 & 0.000 & 103.15 \\
\hline 21 & 12 & 11 & -12.28 & 1.46 & 12.29 & -1.39 & 0.002 & 0.07 \\
\hline 22 & 12 & 13 & -42.29 & -15.31 & 42.32 & 16.20 & 0.033 & 0.89 \\
\hline 23 & 13 & 14 & 127.48 & -3.51 & -127.33 & -12.11 & 0.146 & 1.64 \\
\hline 24 & 14 & 15 & 0.00 & 0.00 & 0.00 & 0.00 & 0.000 & 0.00 \\
\hline 25 & 15 & 16 & -280.00 & -123.00 & 280.77 & 112.64 & 0.773 & 8.07 \\
\hline 26 & 16 & 17 & 214.10 & -92.92 & -213.76 & 82.52 & 0.341 & 4.34 \\
\hline 27 & 16 & 19 & -451.47 & -14.50 & 454.46 & 17.42 & 2.988 & 36.42 \\
\hline 28 & 16 & 21 & -329.71 & 41.95 & 330.53 & -55.83 & 0.820 & 13.83 \\
\hline 29 & 16 & 24 & -42.70 & -79.48 & 42.72 & 72.44 & 0.021 & 0.41 \\
\hline 30 & 17 & 18 & 118.09 & -3.51 & -118.00 & -10.00 & 0.089 & 1.04 \\
\hline 31 & 17 & 27 & 135.67 & -39.01 & -135.45 & 6.39 & 0.222 & 2.96 \\
\hline 32 & 19 & 20 & 174.69 & 3.61 & -174.47 & 0.65 & 0.216 & 4.26 \\
\hline 33 & 19 & 33 & -629.14 & -21.03 & 632.00 & 78.96 & 2.856 & 57.93 \\
\hline 34 & 20 & 34 & -505.53 & -103.65 & 508.00 & 153.10 & 2.472 & 49.45 \\
\hline 35 & 21 & 22 & -604.53 & -59.17 & 607.24 & 78.50 & 2.714 & 47.49 \\
\hline 36 & 22 & 23 & 42.76 & 40.27 & -42.73 & -60.33 & 0.024 & 0.38 \\
\hline 37 & 22 & 35 & -650.00 & -118.77 & 650.00 & 177.74 & 0.000 & 58.97 \\
\hline 38 & 23 & 24 & 353.82 & -19.77 & -351.32 & 19.76 & 2.499 & 39.75 \\
\hline 39 & 23 & 36 & -558.58 & -4.49 & 560.00 & 81.49 & 1.415 & 77.00 \\
\hline 40 & 25 & 26 & -46.04 & -6.66 & 46.12 & -53.07 & 0.075 & 0.76 \\
\hline 41 & 25 & 37 & -538.33 & 120.21 & 540.00 & -55.46 & 1.675 & 64.75 \\
\hline 42 & 26 & 27 & 145.88 & 58.48 & -145.55 & -81.89 & 0.327 & 3.43 \\
\hline 43 & 26 & 28 & -140.68 & -9.26 & 141.48 & -69.69 & 0.797 & 8.79 \\
\hline 44 & 26 & 29 & -190.31 & -13.14 & 192.24 & -81.25 & 1.925 & 21.10 \\
\hline 45 & 28 & 29 & -347.48 & 42.09 & 349.03 & -53.07 & 1.554 & 16.76 \\
\hline \multirow[t]{2}{*}{46} & 29 & 38 & -824.77 & 107.42 & 830.00 & -5.42 & 5.231 & 102.00 \\
\hline & & & & & & Total: & 53.436 & 1110.95 \\
\hline
\end{tabular}


Table A48. Results of the mixed AC/DC PF calculations in Case 7-5. DC bus data.

\begin{tabular}{cccc}
\hline \multirow{2}{*}{ Bus DC } & \multicolumn{2}{c}{ Bus Injection } & Total Loss \\
\cline { 2 - 4 } & $\boldsymbol{P}$ & $\boldsymbol{Q}$ & $\boldsymbol{P}$ \\
\hline 1 & -40.00 & -20.00 & 1.25 \\
2 & -46.04 & 74.15 & 1.42 \\
3 & 40.00 & 40.00 & 1.23 \\
4 & 40.00 & 40.00 & 1.24 \\
5 & -127.33 & -12.11 & 1.75 \\
6 & 40.00 & 30.00 & 1.22 \\
7 & 40.00 & 20.00 & 1.2 \\
8 & 40.00 & 40.00 & 1.24 \\
& \multicolumn{3}{c}{ Total: } \\
\hline
\end{tabular}

Table A49. Results of the mixed AC/DC PF calculations in Case 7-5. DC branch data.

\begin{tabular}{|c|c|c|c|c|c|}
\hline \multirow{2}{*}{ Branch } & \multicolumn{3}{|r|}{ From Bus } & \multirow{2}{*}{$\begin{array}{c}\text { To Bus } \\
P\end{array}$} & \multirow{2}{*}{$\begin{array}{c}\text { Power Losses } \\
P\end{array}$} \\
\hline & From & To & $P$ & & \\
\hline 1 & 1 & 2 & -2.20 & 2.20 & 0.00 \\
\hline 2 & 1 & 4 & 40.95 & -40.51 & 0.44 \\
\hline 3 & 2 & 3 & 42.43 & -41.96 & 0.47 \\
\hline 4 & 3 & 4 & 0.73 & -0.73 & 0.00 \\
\hline 5 & 5 & 6 & 41.86 & -41.22 & 0.64 \\
\hline 6 & 5 & 7 & 41.85 & -41.21 & 0.64 \\
\hline 7 & 5 & 8 & 41.87 & -41.23 & 0.64 \\
\hline 8 & 6 & 7 & -0.01 & 0.01 & 0.00 \\
\hline \multirow[t]{2}{*}{9} & 6 & 8 & 0.01 & -0.01 & 0.00 \\
\hline & & & & Total: & 2.83 \\
\hline
\end{tabular}


Table A50. Results of Mixed AC/DC PF calculations in Case 7-6. AC bus data.

\begin{tabular}{|c|c|c|c|c|c|c|}
\hline \multirow{2}{*}{ Bus } & \multicolumn{2}{|c|}{ Voltage } & \multicolumn{2}{|c|}{ Generation } & \multicolumn{2}{|c|}{ Load } \\
\hline & $|V|$ (p.u.) & $\theta\left({ }^{\circ}\right)$ & $P$ & $Q$ & $P$ & $Q$ \\
\hline 1 & 1.049 & -8.267 & - & - & 97.60 & 44.20 \\
\hline 2 & 1.062 & -2.889 & - & - & - & - \\
\hline 3 & 1.066 & -5.034 & - & - & 322.00 & 2.40 \\
\hline 4 & 0.932 & -16.723 & - & - & 500.00 & 184.00 \\
\hline 5 & 0.967 & -12.294 & - & - & - & - \\
\hline 6 & 0.975 & -11.059 & - & - & - & - \\
\hline 7 & 0.965 & -13.409 & - & - & 233.80 & 84.00 \\
\hline 8 & 0.965 & -13.950 & - & - & 522.00 & 176.60 \\
\hline 9 & 1.025 & -12.240 & - & - & 6.50 & -66.60 \\
\hline 10 & 1.002 & -7.176 & - & - & - & - \\
\hline 11 & 0.993 & -8.494 & - & - & - & - \\
\hline 12 & 1.000 & -8.589 & - & - & 8.53 & 88.00 \\
\hline 13 & 1.001 & -7.424 & - & - & - & - \\
\hline 14 & 1.000 & -7.736 & - & - & - & - \\
\hline 15 & 1.03 & -3.037 & - & - & 320.00 & 153.00 \\
\hline 16 & 1.044 & -1.572 & - & - & 329.00 & 32.30 \\
\hline 17 & 1.050 & -2.488 & - & - & - & - \\
\hline 18 & 1.048 & -3.091 & - & - & 158.00 & 30.00 \\
\hline 19 & 1.054 & 3.016 & - & - & - & - \\
\hline 20 & 0.993 & 1.618 & - & - & 680.00 & 103.00 \\
\hline 21 & 1.040 & 0.800 & - & - & 274.00 & 115.00 \\
\hline 22 & 1.054 & 5.205 & - & - & - & - \\
\hline 23 & 1.05 & 5.008 & - & - & 247.50 & 84.60 \\
\hline 24 & 1.048 & -1.452 & - & - & 308.60 & -92.20 \\
\hline 25 & 1.069 & -1.128 & - & - & 224.00 & 47.20 \\
\hline 26 & 1.063 & -1.501 & - & - & 139.00 & 17.00 \\
\hline 27 & 1.052 & -3.084 & - & - & 281.00 & 75.50 \\
\hline 28 & 1.056 & 1.978 & - & - & 206.00 & 27.60 \\
\hline 29 & 1.054 & 4.719 & - & - & 283.50 & 26.90 \\
\hline 30 & 1.050 & -0.506 & 250.00 & 82.91 & - & - \\
\hline 31 & 0.982 & 0.000 & 696.02 & 347.70 & 9.20 & 4.60 \\
\hline 32 & 0.984 & 0.934 & 650.00 & 280.53 & - & - \\
\hline 33 & 0.997 & 8.223 & 632.00 & 80.97 & - & - \\
\hline 34 & 1.012 & 6.803 & 508.00 & 154.03 & - & - \\
\hline 35 & 1.049 & 10.145 & 650.00 & 180.00 & - & - \\
\hline 36 & 1.064 & 12.829 & 560.00 & 82.78 & - & - \\
\hline 37 & 1.028 & 5.600 & 540.00 & -52.18 & - & - \\
\hline 38 & 1.027 & 11.767 & 830.00 & -3.06 & - & - \\
\hline \multirow[t]{2}{*}{39} & 1.030 & -10.916 & 1000.00 & 99.46 & 1104.00 & 250.00 \\
\hline & Total: & & 6316.02 & 1253.14 & 6254.23 & 1387.10 \\
\hline
\end{tabular}


Table A51. Results of Mixed AC/DC PF calculations in Case 7-6. AC branch data.

\begin{tabular}{|c|c|c|c|c|c|c|c|c|}
\hline \multirow{2}{*}{ Branch } & \multirow[b]{2}{*}{ From } & \multirow[b]{2}{*}{ To } & \multicolumn{2}{|c|}{ From Bus Injection } & \multicolumn{2}{|c|}{ To Bus Injection } & \multicolumn{2}{|c|}{ Power Losses } \\
\hline & & & $P$ & $Q$ & $P$ & $Q$ & $P$ & $Q$ \\
\hline 1 & 1 & 2 & -254.22 & -39.12 & 256.28 & -14.61 & 2.056 & 24.14 \\
\hline 2 & 1 & 39 & 202.75 & 34.92 & -202.32 & -105.31 & 0.426 & 10.66 \\
\hline 3 & 2 & 3 & 277.03 & -56.22 & -276.13 & 37.60 & 0.904 & 10.50 \\
\hline 4 & 2 & 25 & -283.31 & 142.35 & 289.70 & -151.10 & 6.384 & 7.84 \\
\hline 5 & 2 & 30 & -250.00 & -71.52 & 250.00 & 82.91 & 0.000 & 11.39 \\
\hline 6 & 3 & 4 & 0.00 & 0.00 & 0.00 & 0.00 & 0.000 & 0.00 \\
\hline 7 & 3 & 18 & 0.00 & 0.00 & 0.00 & 0.00 & 0.000 & 0.00 \\
\hline 8 & 4 & 5 & -556.28 & -204.00 & 559.49 & 243.27 & 3.211 & 51.37 \\
\hline 9 & 4 & 14 & 0.00 & 0.00 & 0.00 & 0.00 & 0.000 & 0.00 \\
\hline 10 & 5 & 6 & -800.66 & -243.89 & 802.15 & 259.24 & 1.496 & 19.45 \\
\hline 11 & 5 & 8 & 241.17 & 0.62 & -240.67 & -7.41 & 0.498 & 6.97 \\
\hline 12 & 6 & 7 & 425.54 & 84.41 & -424.34 & -76.76 & 1.193 & 18.29 \\
\hline 13 & 6 & 11 & -540.87 & -153.36 & 543.18 & 166.98 & 2.311 & 27.07 \\
\hline 14 & 6 & 31 & -686.82 & -190.29 & 686.82 & 343.10 & 0.000 & 152.81 \\
\hline 15 & 7 & 8 & 190.54 & -7.24 & -190.39 & 1.78 & 0.156 & 1.79 \\
\hline 16 & 8 & 9 & -90.94 & -170.96 & 91.73 & 145.68 & 0.785 & 12.39 \\
\hline 17 & 9 & 39 & -98.23 & -79.08 & 98.32 & -45.23 & 0.094 & 2.36 \\
\hline 18 & 10 & 11 & 548.16 & 170.50 & -546.84 & -163.59 & 1.318 & 14.17 \\
\hline 19 & 10 & 13 & 101.84 & 6.52 & -101.80 & -13.39 & 0.042 & 0.45 \\
\hline 20 & 10 & 32 & -650.00 & -177.03 & 650.00 & 280.53 & 0.000 & 103.50 \\
\hline 21 & 12 & 11 & -3.66 & 3.41 & 3.66 & -3.40 & 0.000 & 0.01 \\
\hline 22 & 12 & 13 & -47.06 & -14.10 & 47.10 & 15.16 & 0.039 & 1.06 \\
\hline 23 & 13 & 14 & 54.70 & -1.77 & -54.67 & -15.17 & 0.027 & 0.31 \\
\hline 24 & 14 & 15 & 0.00 & 0.00 & 0.00 & 0.00 & 0.000 & 0.00 \\
\hline 25 & 15 & 16 & -303.49 & -123.00 & 304.38 & 113.91 & 0.891 & 9.30 \\
\hline 26 & 16 & 17 & 190.48 & -88.27 & -190.20 & 77.07 & 0.275 & 3.50 \\
\hline 27 & 16 & 19 & -451.46 & -17.25 & 454.45 & 20.27 & 2.993 & 36.48 \\
\hline 28 & 16 & 21 & -329.70 & 40.04 & 330.52 & -53.88 & 0.819 & 13.83 \\
\hline 29 & 16 & 24 & -42.70 & -80.72 & 42.72 & 73.70 & 0.021 & 0.42 \\
\hline 30 & 17 & 18 & 141.60 & -3.02 & -141.47 & -10.00 & 0.128 & 1.49 \\
\hline 31 & 17 & 27 & 65.09 & -34.05 & -65.04 & -0.74 & 0.053 & 0.71 \\
\hline 32 & 19 & 20 & 174.69 & 2.73 & -174.47 & 1.53 & 0.216 & 4.26 \\
\hline 33 & 19 & 33 & -629.14 & -23.00 & 632.00 & 80.97 & 2.858 & 57.97 \\
\hline 34 & 20 & 34 & -505.53 & -104.53 & 508.00 & 154.03 & 2.475 & 49.50 \\
\hline 35 & 21 & 22 & -604.52 & -61.12 & 607.24 & 80.55 & 2.718 & 47.57 \\
\hline 36 & 22 & 23 & 42.76 & 40.38 & -42.74 & -60.43 & 0.024 & 0.38 \\
\hline 37 & 22 & 35 & -650.00 & -120.93 & 650.00 & 180.00 & 0.000 & 59.07 \\
\hline 38 & 23 & 24 & 353.82 & -18.44 & -351.32 & 18.50 & 2.500 & 39.78 \\
\hline 39 & 23 & 36 & -558.58 & -5.73 & 560.00 & 82.78 & 1.416 & 77.05 \\
\hline 40 & 25 & 26 & 24.63 & -12.96 & -24.61 & -47.17 & 0.025 & 0.26 \\
\hline 41 & 25 & 37 & -538.33 & 116.86 & 540.00 & -52.18 & 1.673 & 64.68 \\
\hline 42 & 26 & 27 & 216.60 & 54.67 & -215.96 & -74.76 & 0.638 & 6.70 \\
\hline 43 & 26 & 28 & -140.69 & -10.32 & 141.49 & -68.53 & 0.796 & 8.78 \\
\hline 44 & 26 & 29 & -190.30 & -14.18 & 192.23 & -80.07 & 1.923 & 21.09 \\
\hline 45 & 28 & 29 & -347.49 & 40.93 & 349.04 & -51.88 & 1.554 & 16.76 \\
\hline \multirow[t]{2}{*}{46} & 29 & 38 & -824.77 & 105.05 & 830.00 & -3.06 & 5.230 & 101.99 \\
\hline & & & & & & Total: & 50.166 & 1098.10 \\
\hline
\end{tabular}


Table A52. Results of the mixed AC/DC PF calculations in Case 7-6. DC bus data.

\begin{tabular}{cccc}
\hline \multirow{2}{*}{ Bus DC } & \multicolumn{2}{c}{ Bus Injection } & Total Loss \\
\cline { 2 - 4 } & $\boldsymbol{P}$ & $\boldsymbol{Q}$ & $\boldsymbol{P}$ \\
\hline 1 & -56.28 & -20.00 & 1.32 \\
2 & -42.19 & 77.31 & 1.42 \\
3 & 45.87 & 40.00 & 1.25 \\
4 & 46.12 & 40.00 & 1.26 \\
5 & -54.67 & -15.17 & 1.29 \\
6 & 16.51 & 30.00 & 1.16 \\
7 & 16.53 & 20.00 & 1.14 \\
8 & 16.49 & 40.00 & 1.19 \\
& Total: & & 10.03 \\
\hline
\end{tabular}

Table A53. Results of the mixed AC/DC PF calculations in Case 7-6. DC branch data.

\begin{tabular}{|c|c|c|c|c|c|}
\hline \multirow{2}{*}{ Branch } & \multicolumn{3}{|r|}{ From Bus } & \multirow{2}{*}{$\begin{array}{c}\text { To Bus } \\
P\end{array}$} & \multirow{2}{*}{$\begin{array}{c}\text { Power Losses } \\
P\end{array}$} \\
\hline & From & To & $P$ & & \\
\hline 1 & 1 & 2 & 5.27 & -5.26 & 0.01 \\
\hline 2 & 1 & 4 & 49.69 & -49.03 & 0.65 \\
\hline 3 & 2 & 3 & 46.03 & -45.47 & 0.56 \\
\hline 4 & 3 & 4 & -1.65 & 1.65 & 0.00 \\
\hline 5 & 5 & 6 & 17.79 & -17.67 & 0.12 \\
\hline 6 & 5 & 7 & 17.79 & -17.67 & 0.12 \\
\hline 7 & 5 & 8 & 17.79 & -17.67 & 0.12 \\
\hline 8 & 6 & 7 & 0.00 & 0.00 & 0.00 \\
\hline \multirow[t]{2}{*}{9} & 6 & 8 & 0.00 & 0.00 & 0.00 \\
\hline & & & & Total: & 1.58 \\
\hline
\end{tabular}

\section{References}

1. Mohammadi, F.; Nazri, G.-A.; Saif, M. A Bidirectional Power Charging Control Strategy for Plug-in Hybrid Electric Vehicles. Sustainability 2019, 11, 4317. [CrossRef]

2. Bahrman, M.P.; Johnson, B.K. The ABCs of HVDC Transmission Technologies. IEEE Power Energy Mag. 2007, 5, 32-44. [CrossRef]

3. Flourentzou, N.; Agelidis, V.G.; Demetriades, G.D. VSC-Based HVDC Power Transmission Systems: An Overview. IEEE Trans. Power Electron. 2009, 24, 592-602. [CrossRef]

4. Mohammadi, F.; Nazri, G.-A.; Saif, M. An Improved Droop-Based Control Strategy for MT-HVDC Systems. Electronics 2020, 9, 87.

5. Xu, L.; Fan, L. Impedance-Based Resonance Analysis in a VSC-HVDC System. IEEE Trans. Power Deliv. 2013, 28, 2209-2216.

6. Xu, L.; Fan, L.; Miao, Z. DC Impedance-Model-Based Resonance Analysis of a VSC-HVDC System. IEEE Trans. Power Deliv. 2015, 30, 1221-1230. [CrossRef]

7. Pandya, K.S.; Joshi, S.K. A Survey of Optimal Power Flow Methods. J. Theor. Appl. Inf. Technol. 2008, 4, 450-458.

8. Frank, S.; Steponavice, I.; Rebennack, S. Optimal Power Flow: A Bibliographic Survey II: Non-Deterministic and Hybrid Methods. Energy Syst. 2012, 3, 259-289. [CrossRef]

9. Mohammadi, F.; Nazri, G.-A.; Saif, M. A New Topology of a Fast Proactive Hybrid DC Circuit Breaker for MT-HVDC Grids. Sustainability 2019, 11, 4493. [CrossRef]

10. Mohammadi, F.; Zheng, C. Stability Analysis of Electric Power System. In Proceedings of the 4th National Conference on Technology in Electrical and Computer Engineering, Tehran, Iran, 27 December 2018.

11. Mohammadi, F.; Nazri, G.-A.; Saif, M. A Fast Fault Detection and Identification Approach in Power Distribution Systems. In Proceedings of the 5th International Conference on Power Generation Systems and Renewable Energy Technologies (PGSRET), Istanbul, Turkey, 26-27 August 2019. 
12. Arrillaga, J.; Bodger, P. Integration of HVDC Links with Fast-Decoupled Load-Flow Solutions. Proc. Inst. Electr. Eng. 1977, 124, 463-468. [CrossRef]

13. Baradar, M.; Ghandhari, M.; van Hertem, D. The Modeling Multi-Terminal VSC-HVDC in Power Flow Calculation using Unified Methodology. In Proceedings of the 2nd IEEE PES International Conference and Exhibition on Innovative Smart Grid Technologies, Manchester, UK, 5-7 December 2011.

14. Baradar, M.; Ghandhari, M. A Multi-Option Unified Power-Flow Approach for Hybrid AC/DC Grids Incorporating Multi-terminal VSC-HVDC. IEEE Trans. Power Syst. 2013, 28, 2376-2383. [CrossRef]

15. El-Hawary, M.E.; Ibrahim, S.T. A New Approach to AC-DC Load Flow Analysis. Electr. Power Syst. Res. 1995, 33, 193-200. [CrossRef]

16. Beerten, J.; van Hertem, D.; Belmans, R. VSC-MTDC Systems with a Distributed DC Voltage Control-A Power Flow Approach. In Proceedings of the 2011 IEEE Trondheim PowerTech, Trondheim, Norway, 19-23 June 2011.

17. Haileselassie, T.M.; Uhlen, K. Power Flow Analysis of Multi-Terminal HVDC Networks. In Proceedings of the 2011 IEEE Trondheim PowerTech, Trondheim, Norway, 19-23 June 2011.

18. Beerten, J.; Cole, S.; Belmans, R. Implementation Aspects of a Sequential AC/DC Power Flow Computation Algorithm for Multi-Terminal VSC-HVDC Systems. In Proceedings of the 9th IET International Conference on AC and DC Power Transmission, London, UK, 19-21 October 2010.

19. Beerten, J.; Cole, S.; Belmans, R. A Sequential AC/DC Power Flow Algorithm for Networks Containing Multi-Terminal VSC-HVDC Systems. In Proceedings of the IEEE PES General Meeting, Providence, RI, USA, 25-29 July 2010.

20. Beerten, J.; Cole, S.; Belmans, R. Generalized Steady-State VSC MTDC Model for Sequential AC/DC Power Flow Algorithms. IEEE Trans. Power Syst. 2012, 27, 821-829. [CrossRef]

21. Gonzalez-Longatt, F.; Roldan, J.; Charalambous, C.A. Power Flow Solution on Multi-Terminal HVDC Systems: Supergrid Case. In Proceedings of the International Conference on Renewable Energies and Power Quality, Santiago de Compostela, Galicia, Spain, 28-30 March 2012.

22. Hendriks, R.L.; Paap, G.C.; Kling, W.L. Control of a Multi-Terminal VSC Transmission Scheme for Interconnecting Offshore Wind Farms. In Proceedings of the European Wind Energy Conference and Exhibition, Milan, Italy, 7-10 May 2007.

23. Yang, Z.; Zhong, H.; Bose, A.; Xia, Q.; Kang, C. Optimal Power Flow in AC-DC Grids with Discrete Control Devices. IEEE Trans. Power Syst. 2018, 33, 1461-1472. 